\title{
The impact of food reformulation on nutrient intakes and health, a systematic review of modelling studies
}

\author{
Carlo Federici ${ }^{1^{*}}$ DD, Patrick Detzel ${ }^{2}$, Francesco Petracca ${ }^{1}$, Livia Dainelli ${ }^{2}$ and Giovanni Fattore ${ }^{1,3}$
}

\begin{abstract}
Background: Unhealthy diet is a risk factor for adverse health outcomes. Reformulation of processed foods has the potential to improve population diet, but evidence of its impact is limited. The purpose of this review was to explore the impact of reformulation on nutrient intakes, health outcomes and quality of life; and to evaluate the quality of modelling studies on reformulation interventions.
\end{abstract}

Methods: A systematic review of peer-reviewed articles published between January 2000 and December 2017 was performed using MEDLINE, ScienceDirect, Embase, Scopus, Cochrane, and the Centre for Reviews and Dissemination of the University of York. Additional studies were identified through informal searches on Google and specialized websites. Only simulation studies modelling the impact of food reformulation on nutrient intakes and health outcomes were included. Included articles were independently extracted by 2 reviewers using a standardized, pre-piloted data form, including a self-developed tool to assess study quality.

Results: A total of 33 studies met the selected inclusion criteria, with 20, 5 and 3 studies addressing sodium, sugar and fats reformulation respectively, and 5 studies addressing multiple nutrients. Evidence on the positive effects of reformulation on consumption and health was stronger for sodium interventions, less conclusive for sugar and fats. Study features were highly heterogeneous including differences in methods, the type of policy implemented, the extent of the reformulation, and the spectrum of targeted foods and nutrients. Nonetheless, partial between-study comparisons show a consistent relationship between percentages reformulated and reductions in individual consumption. Positive results are also shown for health outcomes and quality of life measures, although comparisons across studies are limited by the heterogeneity in model features and reporting. Study quality was often compromised by short time-horizons, disregard of uncertainty and time dependencies, and lack of model validation.

Conclusions: Reformulation models highlight relevant improvements in diets and population health. While models are valuable tools to evaluate reformulation interventions, comparisons are limited by non-homogeneous designs and assumptions. The use of validated models and extensive scenario analyses would improve models' credibility, providing useful insights for policy-makers.

Review Registration: A research protocol was registered within the PROSPERO database (ID number CRD42017057341).

Keywords: Food reformulation, Modelling, Nutrition policies, Public health, Decision-analytical models, Sodium intake, Sugar intake, Fat intake

\footnotetext{
* Correspondence: carlo.federici@unibocconi.it

${ }^{1}$ CeRGAS (Centre for Research on Health and Social Care Management), SDA

Bocconi School of Management, Milan, Italy

Full list of author information is available at the end of the article
} 


\section{Background}

The growing evidence on the detrimental effects of poor diet on health, including cardiovascular diseases, diabetes and some type of cancers [1-5], has stimulated national and international nutrition programs and policies to strive to reduce the intake of sodium, sugar and fat to the recommended limits [6,7]. Such initiatives largely focus on changing individuals' behavior by promoting social marketing campaigns, community-based interventions and/or primary healthcare programs, whereas other possible strategies target the dietary environment by affecting availability and prices of unhealthy foods. More recently, increasing attention has been given to interventions favoring the reformulation of processed foods towards healthier alternatives $[8,9]$. Reformulation is defined as the process of altering a food or beverage product's recipe or composition to improve the product's health profile [10]. These strategies are consistent with the World Health Assembly 2004 report that called for initiatives by the food industry to reduce the content of harmful nutrients in processed foods and increase the introduction of innovative, healthy and nutritious choices. However, the current policy agenda of reformulation strategies exhibits a prevailing focus on the former objective, thus aiming to remove or reduce public health sensitive nutrients from foods, while maintaining the same appearance, use and characteristics such as flavor, texture and shelf life [11]. According to a recent review, 59 out of 83 countries have on-going programs of work with the industry to reduce sodium in processed foods [12]. To a minor extent, similar efforts are now in place to reduce excess intakes of sugar, particularly from sugar sweetened beverages, and fat [13].

However, despite the growing emphasis given to reformulation, evidence of its impact on both nutrient intakes and health outcomes is limited. Several design characteristics may affect the policy impact. These include i) the voluntary or mandatory nature of the program, and its consequences on the adherence from the industry; ii) the breadth of the reformulation in terms of products targeted; iii) the amount and type of nutrients reformulated; and iv) the pace of implementation, including how reduction targets are set scheduled and reached.

Additionally, how consumers might react to reformulated products and how this will affect the overall policy effectiveness remains largely unexplored, especially for sugar and fat reformulations. Indeed, consumers might react to changes in taste and energy density by shifting to other, non-reformulated products, eating more, or increasing the use of additional ingredients (e.g. discretionary salt and sugar).

Due to these features, estimating the effectiveness of current and future reformulation policies is challenging, and likely to be both policy and context specific.

Given to the obvious difficulties of performing experimental designs to measure the impact of nutrition policies on population health [14], the majority of published studies use mathematical models to predict the effects of reformulation on intakes and clinical outcomes [15]. Nonetheless, the extreme flexibility of modelling, and the required assumptions needed to simplify complex nutrition interventions may introduce a considerable variability, thus limiting between-study comparability and even challenging the plausibility of models results for population health [16]. To the authors' best knowledge, only one previous review collected and critically appraised analytical models predicting the effects of several nutrition interventions, including reformulation, on intake and health [14]. In the study, reformulation was found to have positive effects on intake and health outcomes; however, due to the broader scope of the authors' work, detailed considerations of reformulation-specific characteristics were only partially addressed.

Therefore, the objectives of this review are to focus on reformulation studies aimed at reducing the content of harmful nutrients to: i) further explore the impact of reformulation on the intake of target nutrients, as well as on health outcomes and quality of life measures; and ii) critically evaluate the quality of reformulation models, with a focus on the key elements that are specifically relevant to their appraisal.

\section{Methods}

A research protocol was previously defined following the PRISMA-P guidelines [17, 18], and registered within the PROSPERO database (ID number CRD42017057341). Studies were reviewed according to the PRISMA guidelines [19] (Additional file 1).

\section{Search strategy}

Records published between January 2000 and December 2017 were searched in electronic bibliographic databases including Medline (via Web of Science), ScienceDirect, Embase, Scopus, the Cochrane and Cochrane Public Health Group Specialized Register, and the Centre for Reviews and Dissemination of the University of York. The full search strategy was first defined on Web of Science (Additional file 2), and then adapted to the other databases. Grey literature was retrieved through informal searches on Google, and on the following websites: Opengrey (http://www.opengrey.eu/), WHO, CDC, and the FAO websites.

\section{Selection of studies}

Studies were considered eligible if they addressed mandatory or voluntary food reformulation strategies aimed at reducing individual intake of sodium, saturated fatty acids (SFA), trans-fatty acids (TFA), and sugar. For studies addressing sugar intake, all definitions were accepted, including added sugars, total sugars and free 
sugars. Included reformulation strategies were required to be aimed at healthy individuals without any age restriction, and to target foods commonly available in retail stores. On the contrary, initiatives limited to restaurants or specific settings such as hospitals, schools, and workplace, or interventions targeting fortification and improved intake of "healthy" nutrients were considered out of scope.

The primary outcomes of interest were i) changes in individual intakes of target nutrients; ii) effects on health outcomes, including obesity, incidence of cardiovascular diseases (CVD), type 2 diabetes (T2D), life-years gained or reduced mortality; and iii) changes in health-related quality of life measures, i.e. Quality Adjusted Life Years (QALYs) and Disability Adjusted Life Years (DALYs).

Although the types of studies to be included were not set in advance in the research protocol, a focus was later given on mathematical and statistical models, since a first scoping of the literature showed that modelling studies were by far the most frequent study design to assess the effects of reformulation policies on intake and health outcomes. Focusing on model-specific issues for reformulation interven tions was then deemed more relevant than reporting results from a broader set of study designs.

Cost-effectiveness models were included as well. However, cost data were not reported, since such policies are usually cost-saving [20], and the main focus of the present review was to assess the magnitude of the effects on intake and health outcomes, rather than cost-effectiveness. Finally, only studies in English were considered for review.

\section{Data extraction}

One reviewer (CF) defined the search strategy and imported the retrieved records in Endnote (ver. X6), whereas two reviewers (CF and FP) independently performed title and abstract screening, full-text analysis and data extraction. At each stage, discrepancies were resolved by discussion.

A standardized, pre-piloted form was used for data extraction including data on: model type; target population; time horizon of the analysis; target country; mandatory/ voluntary nature of the policy; target nutrients; data sources used in the models and funding sources.

Even if not explicitly stated, interventions where all products consumed/marketed are reformulated were assumed to be likewise mandatory interventions, as it was assumed that no voluntary policy would achieve such degree of pervasiveness across manufacturers.

The effects of the interventions were collected and reported for each causal step from reduction in intake, through its effects on risk factors levels (e.g. blood pressure), to the impact on clinical outcomes and health related quality of life measures. When reported, both absolute and percentage reductions were collected. To improve betwe en-study comparability, estimates of QALYs or DALYs were reported per 100,000 individuals whenever the total size of the population was reported by the original studies. In addition, salt amounts were converted to sodium amounts using a $1 \mathrm{~g} / 400 \mathrm{mg}$ conversion rate.

In addition, when available, further data were extracted on the technical feasibility of the modelled interventions, and on whether potential behavioral changes among consumers had been considered in the studies.

If modelling results were provided separately by population sub-groups (e.g. age or sex), the weighted mean was calculated across estimates.

\section{Quality assessment of studies}

There are no specific tools to assess the quality of modelling studies evaluating population-wide nutrition interventions. Therefore, a self-developed evaluation tool was used, mainly drawing from relevant criteria recommended by the International Society for Pharmacoeconomics and Outcomes Research (ISPOR) Good Practice Task Force [21]. The proposed tool identifies 9 criteria to assess both the scientific quality of the studies (model validation, credible data inputs, uncertainty analysis, transparency and reporting quality), and the pertinence to the research question (objective, scope and relevance). The full list of criteria and the evaluation guide are provided in the Additional file 3.

\section{Results}

After removing duplicates, the literature search identified 22,907 records from bibliographic databases and other sources. After abstract and title screening, 49 records were analyzed full-text and 33 studies were finally included in the review. Reasons for full-text exclusion were documented and are reported in the flow diagram (Fig. 1).

\section{Study characteristics}

Tables 1, 2 and 3 Fig. 2 and the Additional file 4 summarize the main findings and characteristics of the included studies. Overall, studies were heterogeneous in the way interventions and outcomes were modelled and reported, limiting between-study comparisons. As regards the type of nutrient reformulated, 20 studies focused exclusively on sodium reduction (60.6\%) [22-41], followed by 5 studies considering at least 2 different nutrients (15.1\%) [8, 42-45], 5 studies addressing sugar (15.1\%) [46-50] and 3 study addressing fat $(9 \%)[11,51,52]$.

Target foods in the included studies were all processed foods $(n=9)$ [ $27,31,33,37,38,41,45,51,52]$, selected groups of nutrient-dense foods $(n=8)[11,26,34-36$, $43,44,50]$, or single food products $(n=8)[23,24,42$, 46-49]. Four studies provided estimates for more than one item $[8,28-30]$, whereas in 4 studies target foods were not specified since reformulation was modelled directly through its assumed effect on intake [22, 25, 32, 


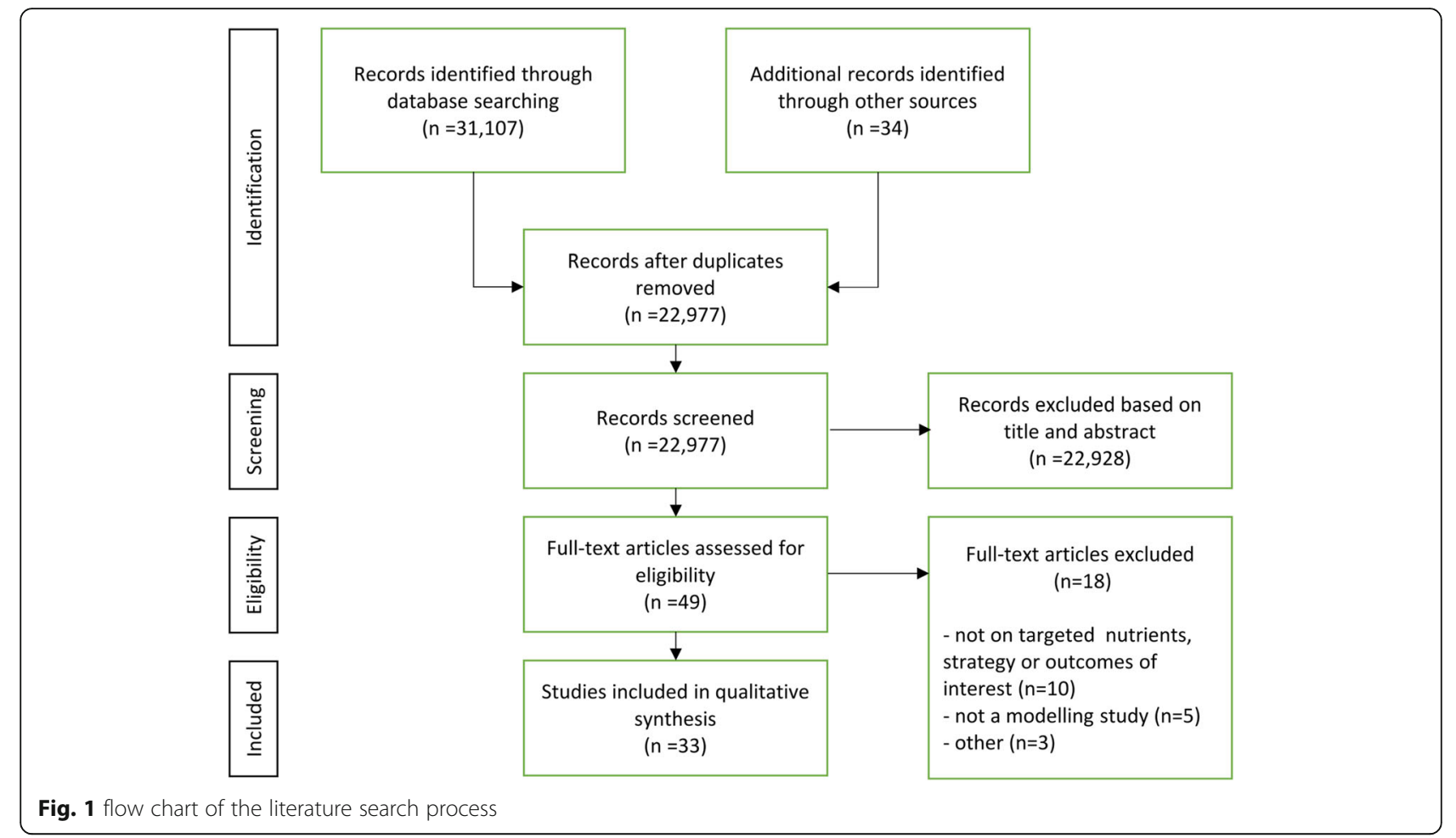

39]. Ten studies simply estimated changes in intake following reformulation $(30.3 \%)[8,11,26,33,42,43,45$, $49,50,53]$, whereas other studies estimated effects on health outcomes $(n=12,36.4 \%)[25,31,35,37,39-41$, $44,46-48,51]$ or health related quality of life measures $(n=11,33.3 \%)[22-24,28-30,32,34,36,38,52]$.

Ten studies used epidemiological models linking changes in nutrients intake to changes in disease incidence and prevalence at an established time $[25,34,35$, 39-41, 44, 47, 52]. These studies include comparative risk assessment models (e.g. WHO Comparative Risk Assessment, or PRIME) [35, 47], potential impact fraction models [34] and other validated models, including DIETRON [44], and IMPACT models [25, 39-41, 51, 52]. Ten studies used cohort Markov models [22, 23, 28-30, $32,36-38$ ], or individual level micro-simulations [31]. Finally, 13 records, accounting for almost $40 \%$ of the included studies, used mathematical-statistical models to estimate the potential change in intake by linking cross-sectional, individual-level consumption data to the nutrient-density of foods before and after reformulation $[8,11,24,26,33,42,43,45,46,48-50,53]$. In the majority of this last group, time was not explicitly considered, since the analysis was limited to a re-assessment of past individual intakes after reformulating all or a specific set of products. In the remaining studies, the time horizon of the analysis was highly variable: 5 studies considered a time ranging from 1 to 6 years [35, 44, 46, 47, 52], 8 studies between 10 and 20 years [25, 31, 37-41, 51] and 9 studies considered the average lifetime of the modelled population [22-24, 28-30, 32, 34, 36].

Strikingly, 30\% of the studies were not clear about what informed the amounts of nutrient reformulated, and whether considerations were made on technical feasibility, or other aspects such as shelf life and palatability.

Most of the studies explored the impact of likewise mandatory policies, i.e. they assumed that all target foods would be reformulated, while only 5 studies performed scenario analysis modelling both voluntary and mandatory reformulation. This was usually done by assuming smaller proportions of reformulated products in the voluntary scenario [34, 41, 43], or by assuming longer implementation times and/or more uncertain effects on intake [22, 29].

Only 2 studies performed scenario analysis to explore the interplay between reformulation policies and consumers' reactions. Roodenburg et al. tested the results of the model against the possibility that consumers maintained the same caloric intake by eating more food [45], whereas Choi et al. considered whether results were robust to the possibility that consumers might add discretionary salt, or switch to more salty alternatives after introducing low-salt reformulations [31].

\section{Interventions aiming to reduce sodium intake}

Reported percentages of sodium reductions in the models varied between 11 and 63\% across different products, focusing particularly on bread, sauces and processed meats (Table 1). 


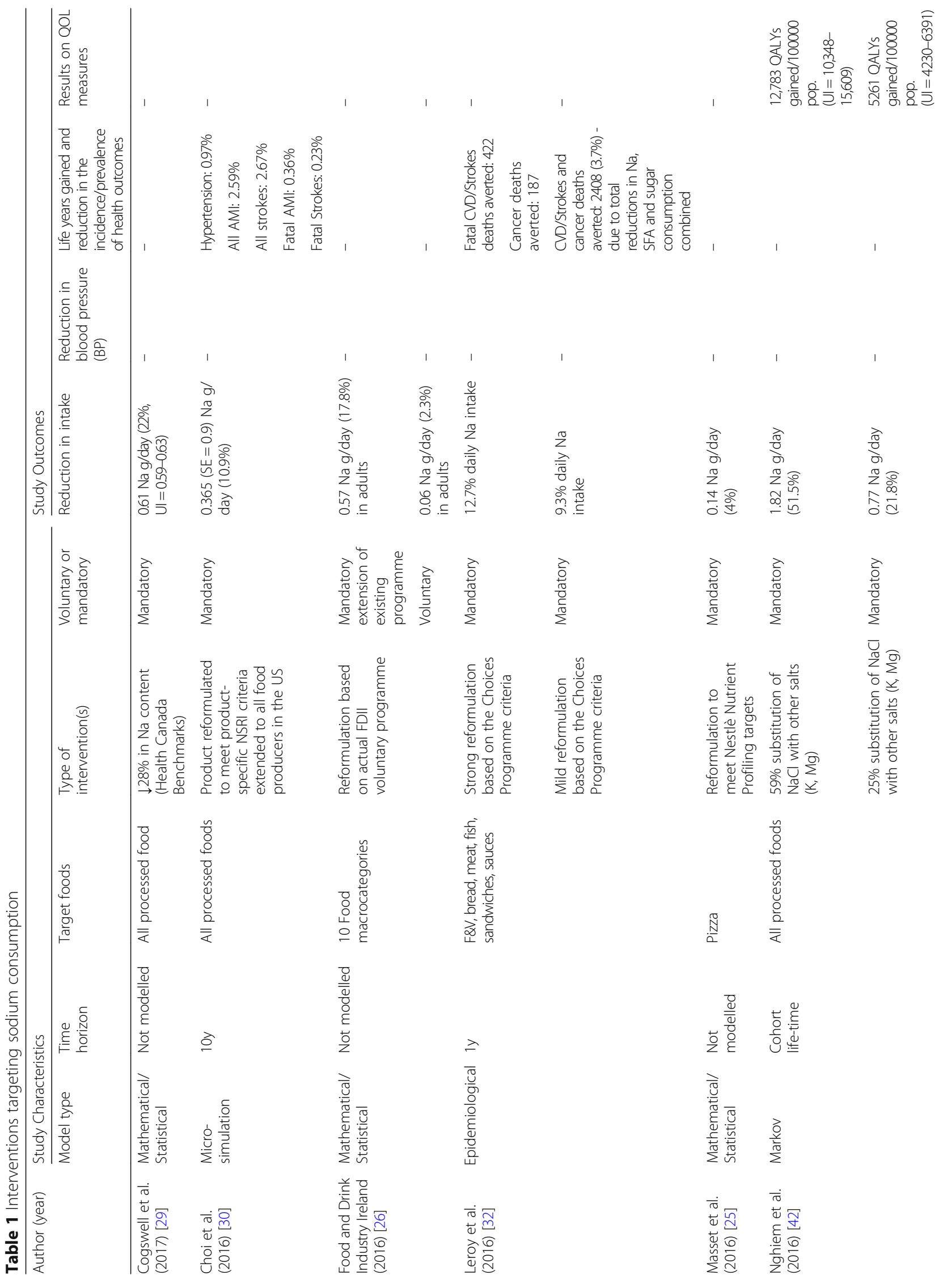




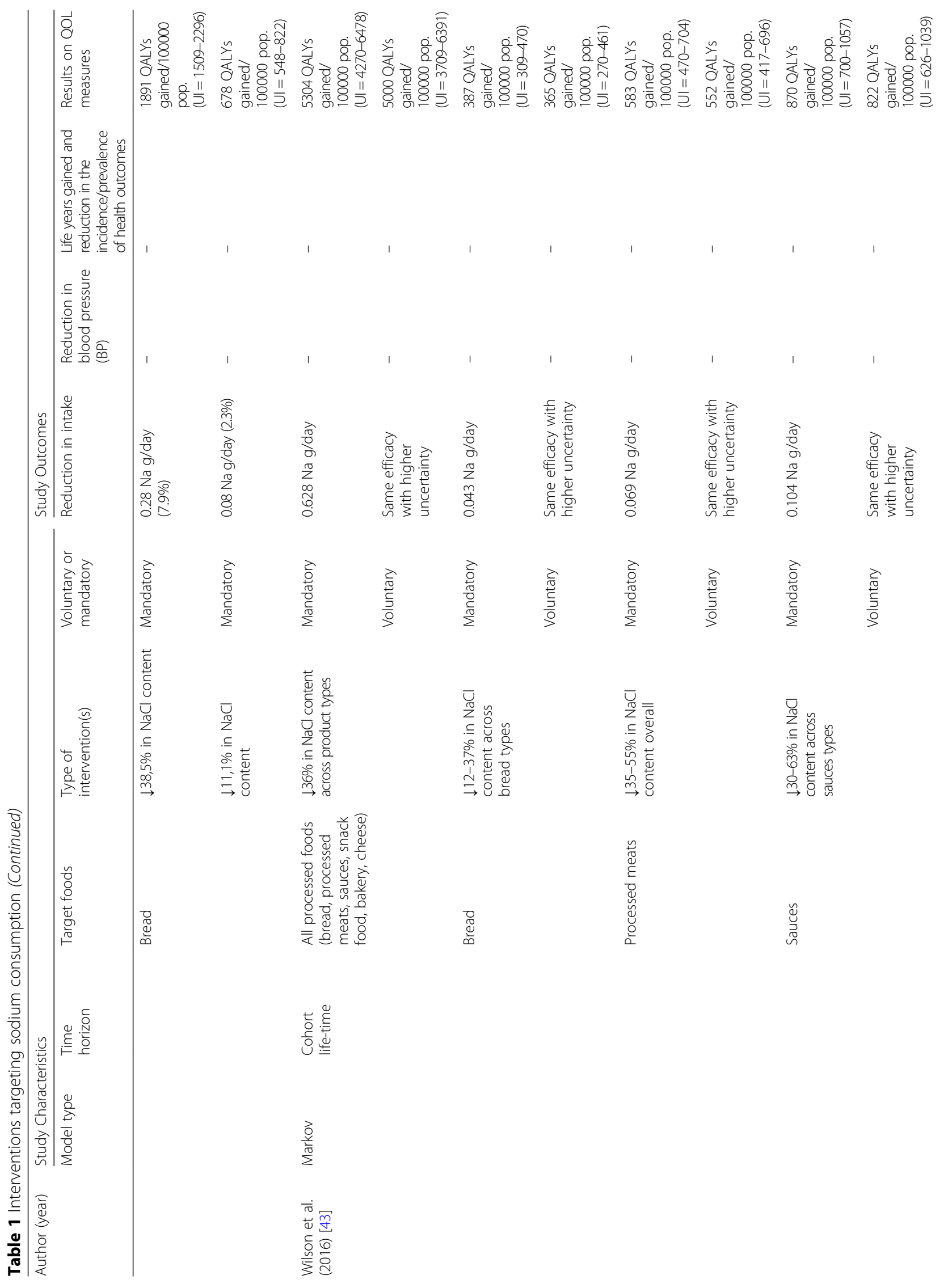




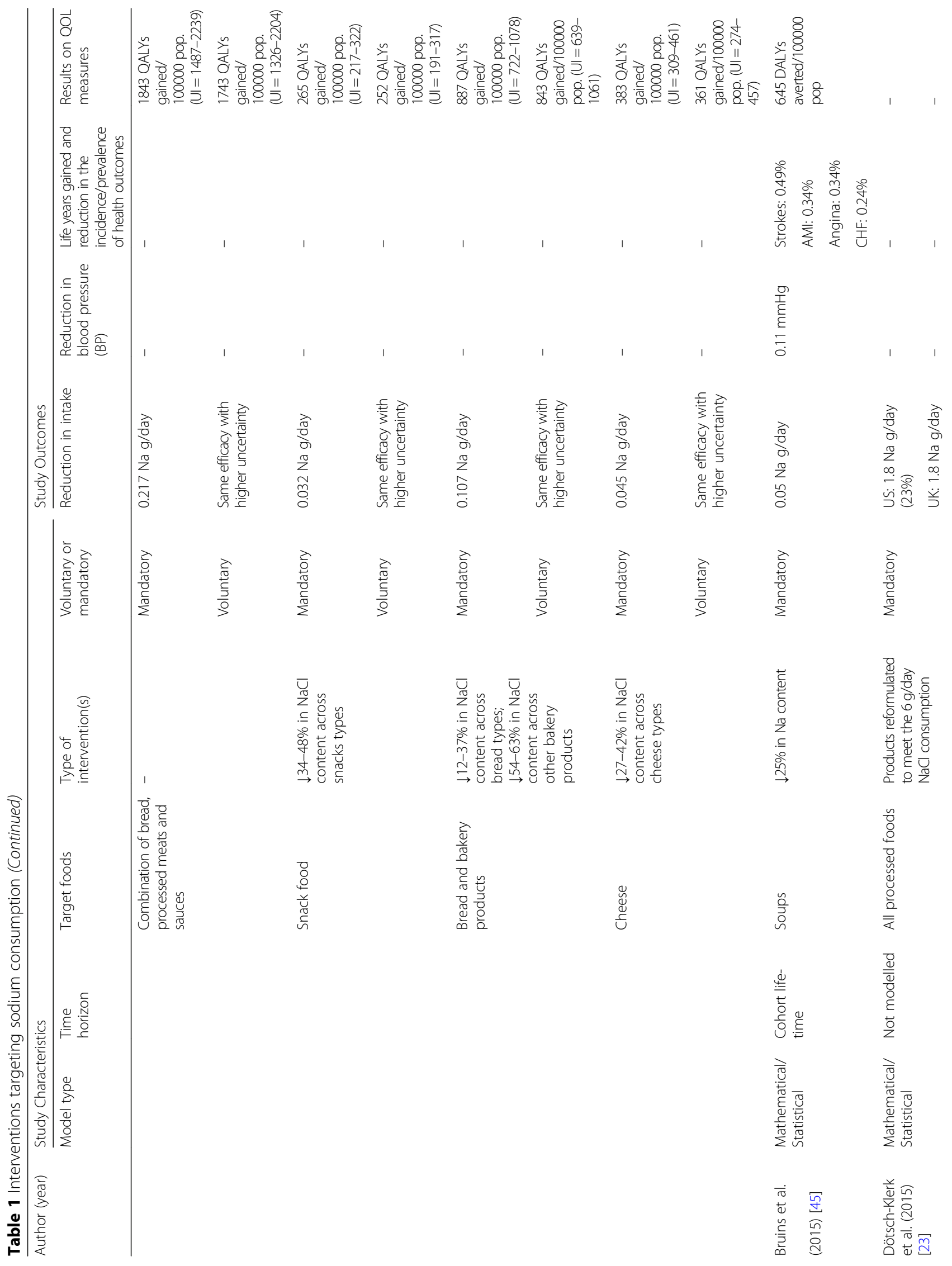




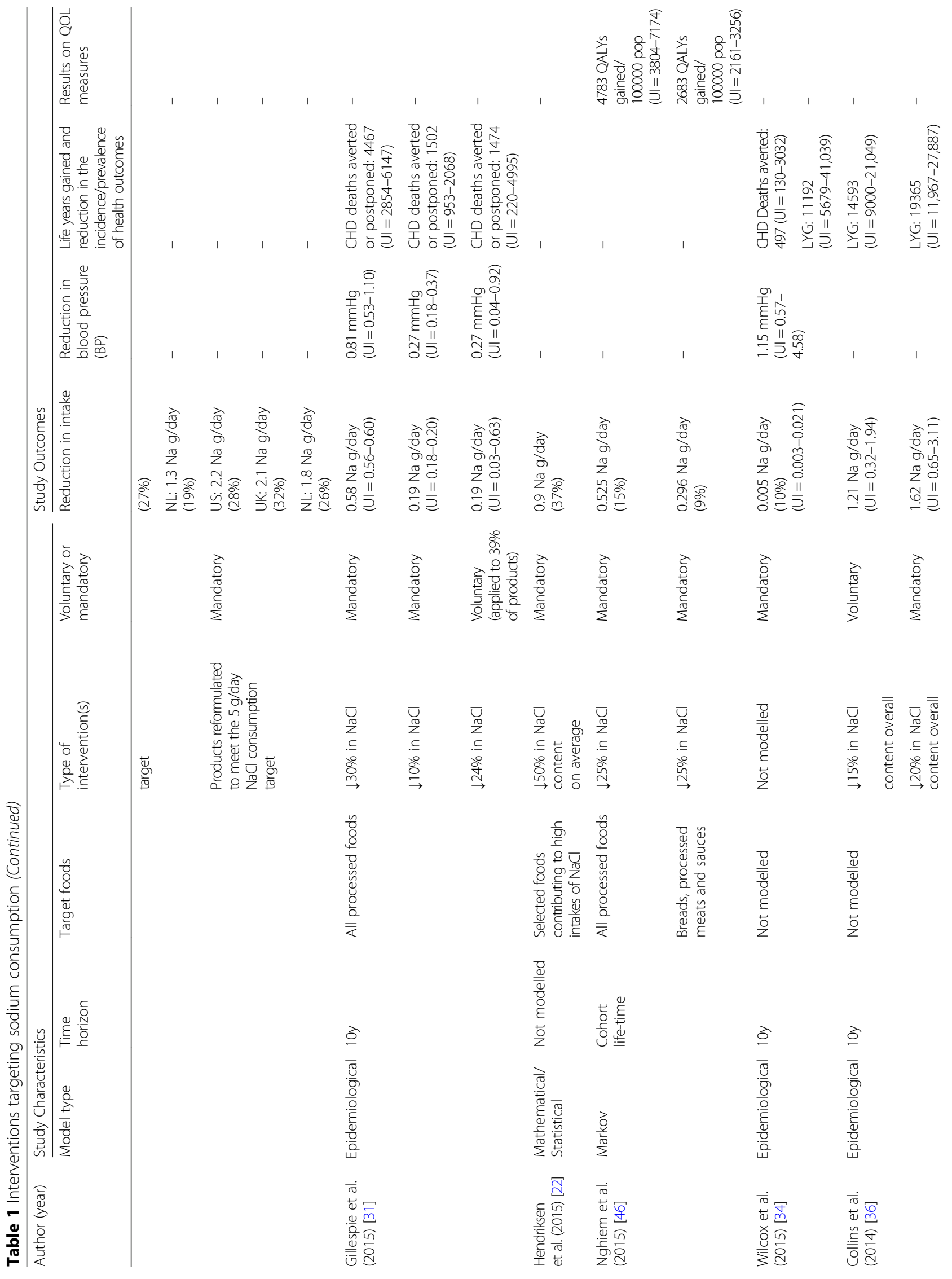




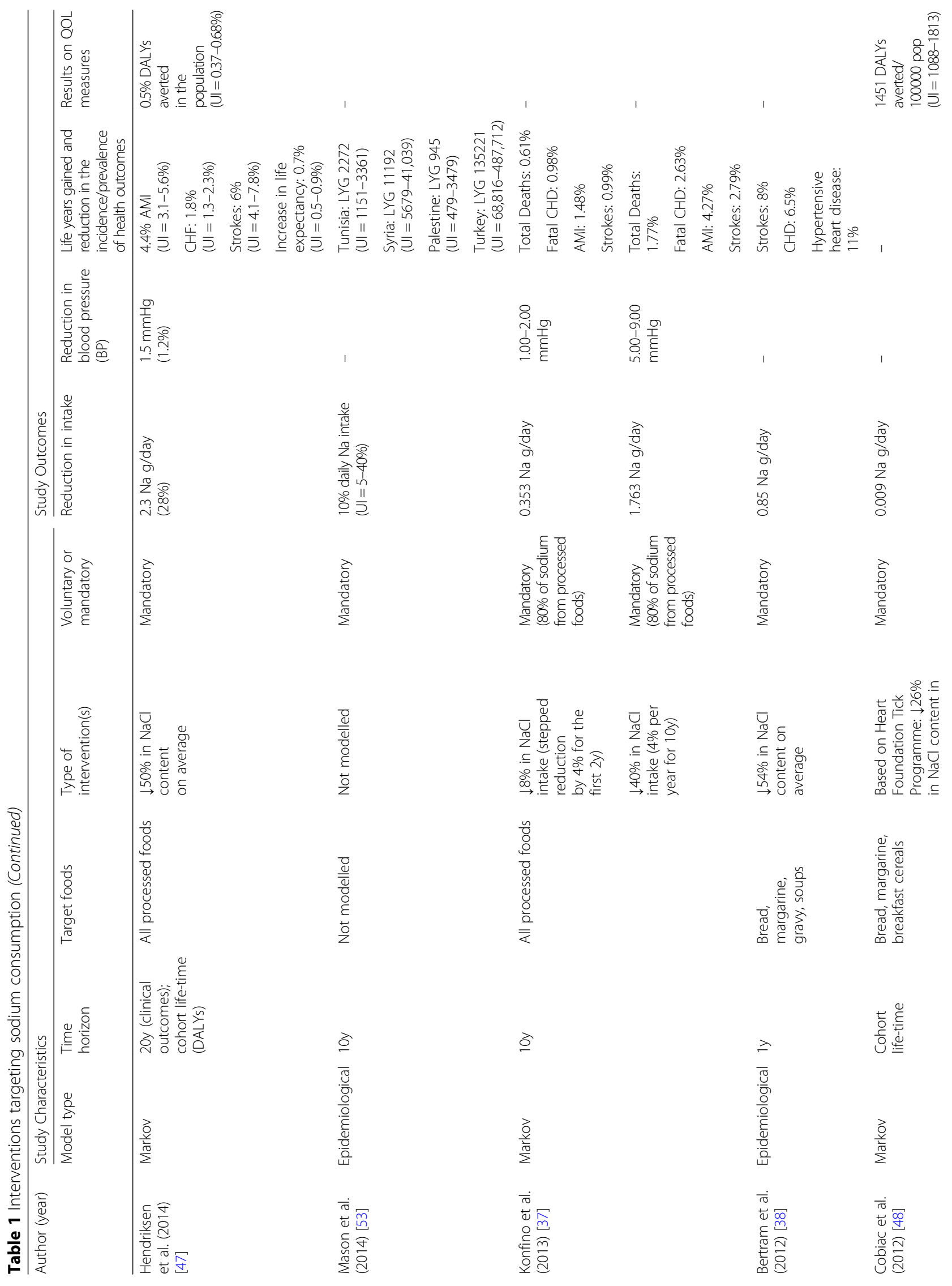




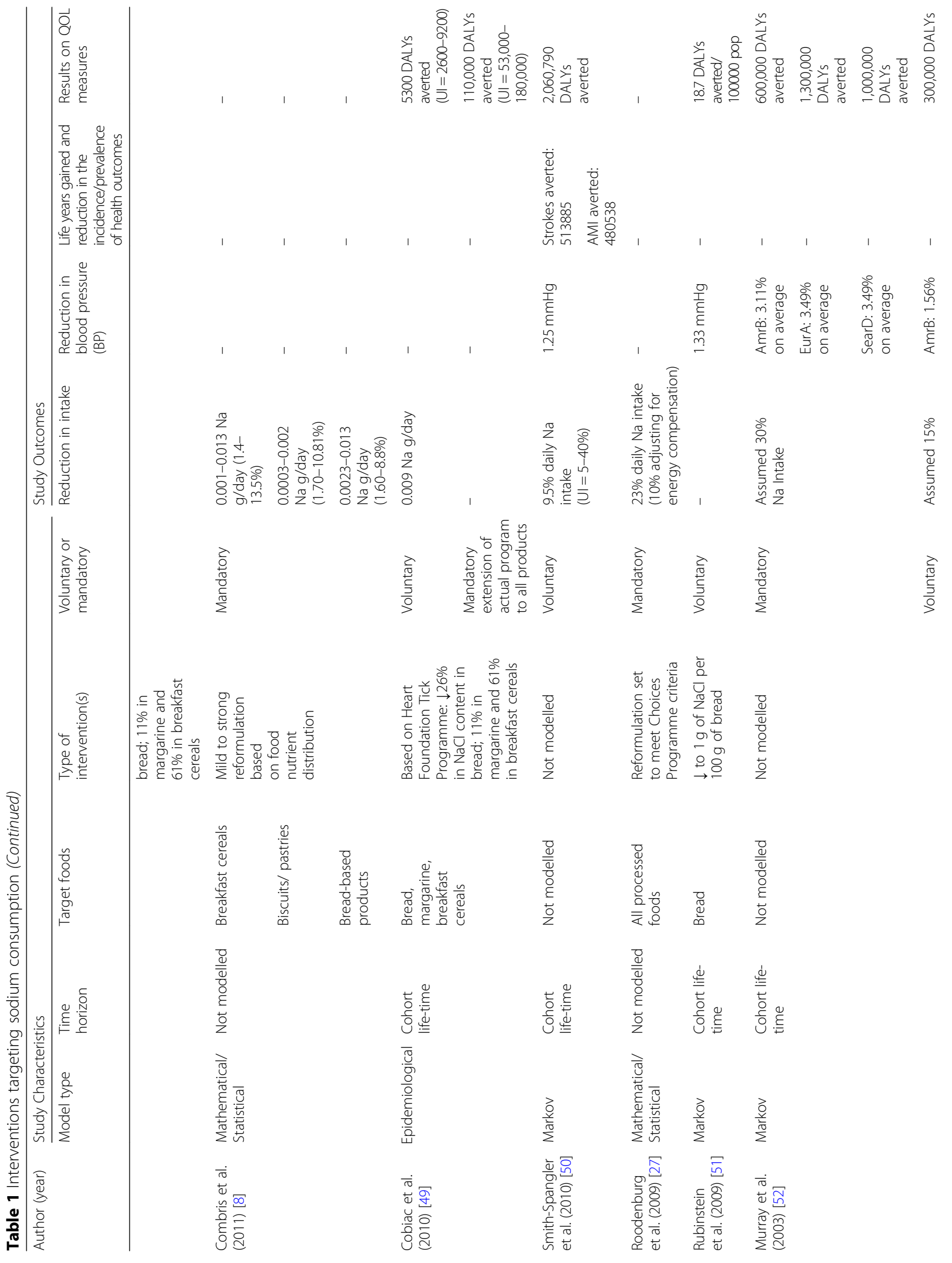




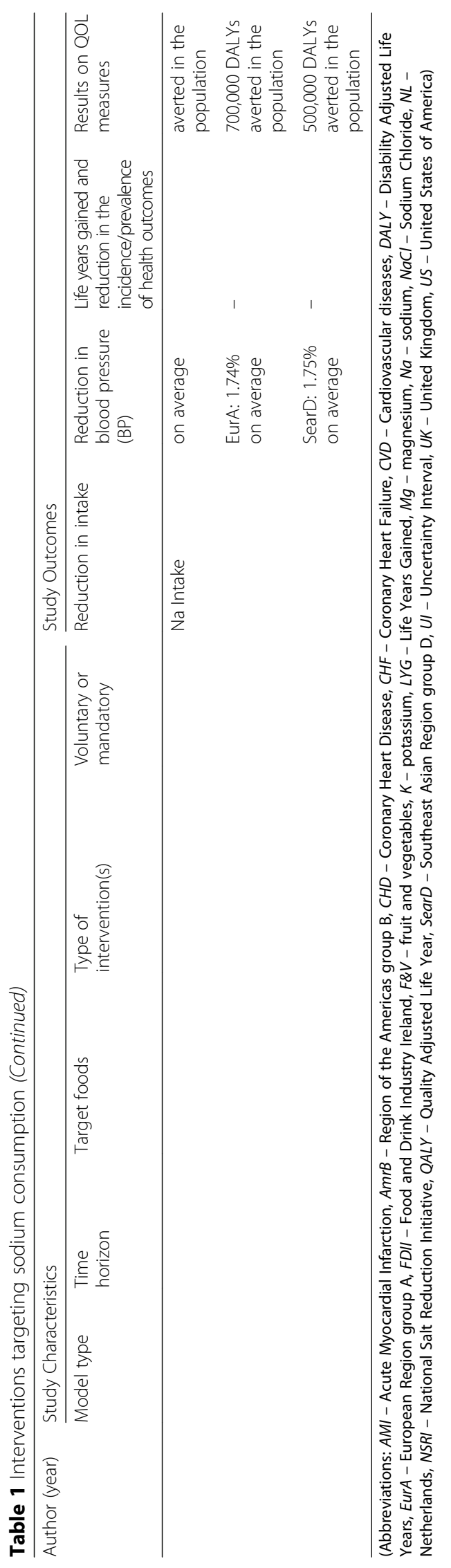


Table $\mathbf{2}$ - Interventions targeting sugar consumption

\begin{tabular}{|c|c|c|c|c|c|c|c|c|}
\hline \multirow[t]{2}{*}{ Author (year) } & \multicolumn{5}{|c|}{ Study Characteristics } & \multicolumn{3}{|l|}{ Study Outcomes } \\
\hline & Model type & $\begin{array}{l}\text { Time } \\
\text { Horizon }\end{array}$ & Target foods & $\begin{array}{l}\text { Type of } \\
\text { intervention(s) }\end{array}$ & $\begin{array}{l}\text { Voluntary/ } \\
\text { mandatory }\end{array}$ & $\begin{array}{l}\text { Reduction in } \\
\text { individual intake }\end{array}$ & $\begin{array}{l}\text { Reduction } \\
\text { in weight }\end{array}$ & $\begin{array}{l}\text { Reduction in } \\
\text { the incidence } \\
\text { of clinical outcomes }\end{array}$ \\
\hline \multirow[t]{4}{*}{$\begin{array}{l}\text { Briggs et al. } \\
\text { (2017) [39] }\end{array}$} & \multirow[t]{4}{*}{ Epidemiological } & \multirow[t]{4}{*}{$1 y$} & \multirow[t]{4}{*}{$\begin{array}{l}\text { High and mid- } \\
\text { sugar drinks }\end{array}$} & \multirow[t]{2}{*}{$\begin{array}{l}\downarrow 30 \% \text { in sugar } \\
\text { for high-sugar } \\
\text { drinks; } 15 \% \text { for } \\
\text { mid-sugar drinks }\end{array}$} & \multirow[t]{2}{*}{ Mandatory } & \multirow[t]{2}{*}{$\begin{array}{l}5.38 \text { sugar g/ } \\
\text { day }(27.5 \%, \mathrm{Ul} \\
=4.19-5.76)- \\
\text { i.e. about } 21 \\
\text { Kcal/day }\end{array}$} & \multirow[t]{2}{*}{-} & $\begin{array}{l}\text { T2D incidence: } 31.1 \\
\text { per 100,000 } \\
\text { persons }(\mathrm{UI}= \\
11-53)\end{array}$ \\
\hline & & & & & & & & $\begin{array}{l}\text { Obesity prevalence: } \\
0.9 \%(U \mathrm{I}=0.3-19 \%)\end{array}$ \\
\hline & & & & \multirow{2}{*}{$\begin{array}{l}\downarrow 5 \% \text { in sugar } \\
\text { content for } \\
\text { both high and } \\
\text { mid-sugar drinks }\end{array}$} & \multirow[t]{2}{*}{ Mandatory } & \multirow{2}{*}{$\begin{array}{l}0.98 \text { sugar g/ } \\
\text { day }(5 \%, \mathrm{UI}= \\
0.92-1.05) \text { - i.e. } \\
\text { about } 3.92 \mathrm{Kcal} / \\
\text { day }\end{array}$} & \multirow[t]{2}{*}{ - } & $\begin{array}{l}\text { T2D incidence: } 5.8 \\
\text { per } 100,000 \text { persons } \\
(U I=2-10)\end{array}$ \\
\hline & & & & & & & & $\begin{array}{l}\text { Obesity prevalence: } \\
0.2 \%(\mathrm{UI}=0.09-4 \%)\end{array}$ \\
\hline \multirow[t]{6}{*}{$\begin{array}{l}\text { Yeung et al. } \\
\text { (2017) [28] }\end{array}$} & \multirow[t]{6}{*}{$\begin{array}{l}\text { Mathematical/ } \\
\text { statistical }\end{array}$} & \multirow[t]{6}{*}{$\begin{array}{l}\text { Not } \\
\text { modelled }\end{array}$} & \multirow{6}{*}{$\begin{array}{l}\text { Selected foods } \\
\text { with at least } 5 \mathrm{~g} \\
\text { of added sugars/ } \\
100 \mathrm{~g}\end{array}$} & \multirow[t]{2}{*}{$\begin{array}{l}\downarrow 10 \% \text { in added } \\
\text { sugar }\end{array}$} & \multirow[t]{2}{*}{ Mandatory } & $\begin{array}{l}10.75 \mathrm{Kcal} / \text { day } \\
(\mathrm{SE}=36,0.52 \%) \\
\text { in } 2-16 \mathrm{y}\end{array}$ & \multirow[t]{2}{*}{-} & \multirow[t]{2}{*}{-} \\
\hline & & & & & & $\begin{array}{l}4.62 \text { added } \\
\text { sugar } \mathrm{g} / \text { day (SE } \\
=2.9,7.69 \%) \text { in } \\
2-16 y\end{array}$ & & \\
\hline & & & & \multirow[t]{2}{*}{$\begin{array}{l}\downarrow 15 \% \text { in added } \\
\text { sugar }\end{array}$} & \multirow[t]{2}{*}{ Mandatory } & $\begin{array}{l}16.25 \mathrm{Kcal} / \text { day } \\
(\mathrm{SE}=55,0.79 \%) \\
\text { in } 2-16 \mathrm{y}\end{array}$ & \multirow[t]{2}{*}{-} & \multirow[t]{2}{*}{-} \\
\hline & & & & & & $\begin{array}{l}6.97 \text { added } \\
\text { sugar g/day (SE } \\
=4.47,11.59 \%) \\
\text { in } 2-16 y\end{array}$ & & \\
\hline & & & & \multirow[t]{2}{*}{$\begin{array}{l}\downarrow 25 \% \text { in added } \\
\text { sugar }\end{array}$} & \multirow[t]{2}{*}{ Mandatory } & $\begin{array}{l}27.24 \mathrm{Kcal} / \mathrm{day} \\
(\mathrm{SE}=92,1.34 \%) \\
\text { in } 2-16 \mathrm{y}\end{array}$ & \multirow[t]{2}{*}{-} & \multirow[t]{2}{*}{-} \\
\hline & & & & & & $\begin{array}{l}11.73 \text { added } \\
\text { sugar } \mathrm{g} / \text { day (SE } \\
=7.5,19.5 \% \text { ) in } \\
2-16 y\end{array}$ & & \\
\hline \multirow[t]{2}{*}{$\begin{array}{l}\text { Food and } \\
\text { Drink Industry } \\
\text { Ireland (2016) } \\
\text { [26] }\end{array}$} & \multirow[t]{2}{*}{$\begin{array}{l}\text { Mathematical/ } \\
\text { statistical }\end{array}$} & \multirow[t]{2}{*}{$\begin{array}{l}\text { Not } \\
\text { modelled }\end{array}$} & \multirow[t]{2}{*}{$\begin{array}{l}10 \text { Food } \\
\text { macrocategories }\end{array}$} & $\begin{array}{l}\text { Reformulation } \\
\text { based on actual } \\
\text { FDll voluntary } \\
\text { programme }\end{array}$ & $\begin{array}{l}\text { Mandatory } \\
\text { extension } \\
\text { of existing } \\
\text { programme }\end{array}$ & $\begin{array}{l}1.02 \text { sugar } \mathrm{g} / \\
\text { day }(1.12 \%) \text { in } \\
\text { adults }\end{array}$ & - & - \\
\hline & & & & & Voluntary & $\begin{array}{l}0.27 \text { sugar g/ } \\
\text { day }(0.30 \%) \text { in } \\
\text { adults }\end{array}$ & - & - \\
\hline $\begin{array}{l}\text { Leroy et al. } \\
\text { (2016) [32] }\end{array}$ & Epidemiological & 1y & $\begin{array}{l}\text { F\&V, bread, } \\
\text { meat, fish, }\end{array}$ & $\begin{array}{l}\text { Strong } \\
\text { reformulation }\end{array}$ & Mandatory & $\begin{array}{l}14.4 \% \text { daily } \\
\text { sugar intake }\end{array}$ & - & $\begin{array}{l}\text { Fatal CVD/Strokes } \\
\text { deaths averted: } 421\end{array}$ \\
\hline & & & $\begin{array}{l}\text { sandwiches, } \\
\text { sauces }\end{array}$ & $\begin{array}{l}\text { based on the } \\
\text { Choices } \\
\text { Programme criteria }\end{array}$ & & & & $\begin{array}{l}\text { Cancer deaths } \\
\text { averted: } 324\end{array}$ \\
\hline & & & & $\begin{array}{l}\text { Mild reformulation } \\
\text { based on the } \\
\text { Choices Programme } \\
\text { criteria }\end{array}$ & Mandatory & $\begin{array}{l}4.6 \% \text { daily sugar } \\
\text { intake }\end{array}$ & - & $\begin{array}{l}\text { CVD/Strokes and } \\
\text { Cancer deaths } \\
\text { averted: } 2408 \text { (3.7\%) } \\
\text { - due to total } \\
\text { reductions in } \mathrm{Na} \text {, } \\
\text { SFA and sugar } \\
\text { consumption } \\
\text { combined }\end{array}$ \\
\hline $\begin{array}{l}\text { Ma et al. } \\
\text { (2016) [40] }\end{array}$ & $\begin{array}{l}\text { Mathematical/ } \\
\text { statistical }\end{array}$ & $5 y$ & $\begin{array}{l}\text { Sugar } \\
\text { sweetened }\end{array}$ & $\begin{array}{l}\downarrow 40 \% \text { in added } \\
\text { sugar content }(9.7 \%\end{array}$ & Mandatory & $\begin{array}{l}38.4 \mathrm{Kcal} / \mathrm{day} \\
(\mathrm{UI}=36.3-40.7)\end{array}$ & $\begin{array}{l}1.2 \mathrm{~kg}(\mathrm{Ul} \\
=1.12-\end{array}$ & $\begin{array}{l}\text { BMI reduction: } 0.42 \\
\mathrm{~kg} / \mathrm{m}^{2}(1.5 \%)\end{array}$ \\
\hline & & & $\begin{array}{l}\text { beverages (with } \\
\text { juices) }\end{array}$ & $\begin{array}{l}\text { per year over } 5 \\
\text { years) }\end{array}$ & & & & $\begin{array}{l}\text { Overweight } \\
\text { prevalence: } 1 \%\end{array}$ \\
\hline
\end{tabular}


Table 2 - Interventions targeting sugar consumption (Continued)

\begin{tabular}{|c|c|c|c|c|c|c|c|c|}
\hline \multirow[t]{2}{*}{ Author (year) } & \multicolumn{5}{|c|}{ Study Characteristics } & \multicolumn{3}{|l|}{ Study Outcomes } \\
\hline & Model type & $\begin{array}{l}\text { Time } \\
\text { Horizon }\end{array}$ & Target foods & $\begin{array}{l}\text { Type of } \\
\text { intervention(s) }\end{array}$ & $\begin{array}{l}\text { Voluntary/ } \\
\text { mandatory }\end{array}$ & $\begin{array}{l}\text { Reduction in } \\
\text { individual intake }\end{array}$ & $\begin{array}{l}\text { Reduction } \\
\text { in weight }\end{array}$ & $\begin{array}{l}\text { Reduction in } \\
\text { the incidence } \\
\text { of clinical outcomes }\end{array}$ \\
\hline & & & & & & & & $\begin{array}{l}\text { Obesity } \\
\text { prevalence: } \\
2.1 \%\end{array}$ \\
\hline & & & & & & & & $\begin{array}{l}\text { T2D incidence: } \\
\text { 274000-309,000 } \\
\text { cases averted }\end{array}$ \\
\hline & & & $\begin{array}{l}\text { Sugar } \\
\text { sweetened } \\
\text { beverages }\end{array}$ & & & $\begin{array}{l}31.0 \mathrm{Kcal} / \mathrm{day} \\
(\mathrm{UI}=28.6-33.7)\end{array}$ & $\begin{array}{l}0.96 \mathrm{~kg} \\
(\mathrm{UI}= \\
0.88-1.04)\end{array}$ & $\begin{array}{l}\text { BMI reduction: } \\
0.34 \mathrm{~kg} / \mathrm{m}^{2} \\
(1.2 \%)\end{array}$ \\
\hline & & & (without juices) & & & & & $\begin{array}{l}\text { Overweight } \\
\text { prevalence: } \\
0.7 \%\end{array}$ \\
\hline & & & & & & & & $\begin{array}{l}\text { Obesity } \\
\text { prevalence: } \\
1.7 \%\end{array}$ \\
\hline & & & & & & & & $\begin{array}{l}\text { T2D incidence } \\
221,000-250,000 \\
\text { cases averted }\end{array}$ \\
\hline $\begin{array}{l}\text { Masset et al. } \\
\text { (2016) [25] }\end{array}$ & $\begin{array}{l}\text { Mathematical/ } \\
\text { statistical }\end{array}$ & $\begin{array}{l}\text { Not } \\
\text { modelled }\end{array}$ & Pizza & $\begin{array}{l}\text { Reformulation to } \\
\text { meet Nestlè } \\
\text { Nutrient Profiling } \\
\text { targets }\end{array}$ & Mandatory & $\begin{array}{l}0.1 \text { sugar g/day } \\
(0.1 \%)\end{array}$ & - & - \\
\hline \multirow[t]{3}{*}{$\begin{array}{l}\text { Combris et al. } \\
(2011)[8]\end{array}$} & \multirow[t]{3}{*}{$\begin{array}{l}\text { Mathematical/ } \\
\text { statistical }\end{array}$} & \multirow[t]{3}{*}{$\begin{array}{l}\text { Not } \\
\text { modelled }\end{array}$} & Breakfast cereals & \multirow{3}{*}{$\begin{array}{l}\text { Mild to strong } \\
\text { reformulation based } \\
\text { on food nutrient } \\
\text { distribution }\end{array}$} & \multirow[t]{3}{*}{ Mandatory } & $\begin{array}{l}0.125-0.278 \\
\text { sugar g/day } \\
(1.80-4 \%)\end{array}$ & - & - \\
\hline & & & Biscuits/ pastries & & & $\begin{array}{l}0.006-0.068 \\
\text { sugar g/day } \\
(0.30-3.5 \%)\end{array}$ & - & - \\
\hline & & & $\begin{array}{l}\text { Bread-based } \\
\text { products }\end{array}$ & & & $\begin{array}{l}0.058-0.288 \\
\text { sugar g/day } \\
(2.80-13.9 \%)\end{array}$ & - & - \\
\hline \multirow{2}{*}{$\begin{array}{l}\text { Hendriksen } \\
\text { et al. (2011) } \\
\text { [41] }\end{array}$} & \multirow[t]{2}{*}{$\begin{array}{l}\text { Mathematical/ } \\
\text { statistical }\end{array}$} & \multirow[t]{2}{*}{$\begin{array}{l}\text { Not } \\
\text { modelled }\end{array}$} & \multirow[t]{2}{*}{$\begin{array}{l}\text { Carbonated soft } \\
\text { drinks }\end{array}$} & \multirow{2}{*}{$\begin{array}{l}100 \% \text { substitution } \\
\text { of sugar with } \\
\text { intense sweeteners }\end{array}$} & \multirow[t]{2}{*}{ Mandatory } & \multirow[t]{2}{*}{$80.5 \mathrm{Kcal} /$ day } & \multirow[t]{2}{*}{$3.55 \mathrm{~kg}$} & $\begin{array}{l}\text { Obesity } \\
\text { prevalence: } 4 \%\end{array}$ \\
\hline & & & & & & & & $\begin{array}{l}\text { BMI reduction: } \\
1.5 \mathrm{~kg} / \mathrm{m}^{2}\end{array}$ \\
\hline $\begin{array}{l}\text { Roodenburg } \\
\text { et al. (2009) } \\
{[27]}\end{array}$ & $\begin{array}{l}\text { Mathematical/ } \\
\text { statistical }\end{array}$ & $\begin{array}{l}\text { Not } \\
\text { modelled }\end{array}$ & $\begin{array}{l}\text { All processed } \\
\text { foods }\end{array}$ & $\begin{array}{l}\text { Reformulation set } \\
\text { to meet Choices } \\
\text { Programme criteria }\end{array}$ & Mandatory & $\begin{array}{l}37 \% \text { daily sugar } \\
\text { intake ( } 29 \% \\
\text { adjusting for } \\
\text { energy } \\
\text { compensation) }\end{array}$ & - & - \\
\hline $\begin{array}{l}\text { Husøy et al. } \\
\text { (2008) [24] }\end{array}$ & $\begin{array}{l}\text { Mathematical/ } \\
\text { statistical }\end{array}$ & $\begin{array}{l}\text { Not } \\
\text { modelled }\end{array}$ & $\begin{array}{l}\text { Carbonated soft } \\
\text { drinks }\end{array}$ & $\begin{array}{l}100 \% \text { substitution } \\
\text { of sugar with } \\
\text { intense sweeteners }\end{array}$ & Mandatory & $\begin{array}{l}36.5 \% \text { energy } \\
\text { intake }\end{array}$ & - & - \\
\hline
\end{tabular}

Abbreviations: BMI - Body Mass Index; CVD - Cardiovascular diseases; FDII - Food and Drink Industry Ireland; F\&V - fruit and vegetables; SE - Standard Error; T2D - Type 2 diabetes; UI - Uncertainty Interval

Studies reporting absolute reductions in sodium intake showed effects ranging from 0.009 to $1.82 \mathrm{~g} /$ day per person, mainly depending on the amount of nutrients reformulated, the spectrum of targeted foods and scenario studied [30,36]. Overall, a certain degree of consistency was found across studies, with higher percentages of sodium reformulated leading to higher reductions in sodium intake, which in addition tend to be more marked for interventions addressing all processed foods compared to narrower sets of products (Fig. 3, panel A).

Studies estimating the effects on health outcomes showed a percentage reduction in CVD-related mortality in a range between 0.6 and $1.7 \%$ [31, 37], stroke incidence between 0.5 and $8 \%[24,31,35,37,38]$, and Acute Myocardial infarction (AMI) incidence between 0.3 and $4.4 \%[24,31,37,38]$. 
Table 3 - Interventions targeting fat consumption

\begin{tabular}{|c|c|c|c|c|c|c|c|c|}
\hline \multirow[t]{2}{*}{ Author (year) } & \multicolumn{5}{|c|}{ Study Characteristics } & \multicolumn{3}{|l|}{ Study Outcomes } \\
\hline & Model type & $\begin{array}{l}\text { Time } \\
\text { horizon }\end{array}$ & Target foods & $\begin{array}{l}\text { Type of } \\
\text { intervention(s) }\end{array}$ & $\begin{array}{l}\text { Voluntary/ } \\
\text { mandatory }\end{array}$ & $\begin{array}{l}\text { Reduction in } \\
\text { individual } \\
\text { intake }\end{array}$ & $\begin{array}{l}\text { Reduction/increase } \\
\text { in the incidence of } \\
\text { clinical outcomes }\end{array}$ & $\begin{array}{l}\text { Results } \\
\text { on QOL } \\
\text { measures }\end{array}$ \\
\hline \multirow[t]{2}{*}{$\begin{array}{l}\text { Food and } \\
\text { Drink Industry } \\
\text { Ireland (2016) } \\
\text { [26] }\end{array}$} & \multirow[t]{2}{*}{$\begin{array}{l}\text { Mathematical/ } \\
\text { statistical }\end{array}$} & \multirow[t]{2}{*}{$\begin{array}{l}\text { Not } \\
\text { modelled }\end{array}$} & \multirow[t]{2}{*}{$\begin{array}{l}10 \text { Food } \\
\text { macrocategories }\end{array}$} & \multirow[t]{2}{*}{$\begin{array}{l}\text { Reformulation } \\
\text { based on actual } \\
\text { FDIl voluntary } \\
\text { programme }\end{array}$} & $\begin{array}{l}\text { Mandatory } \\
\text { extension } \\
\text { of existing } \\
\text { programme }\end{array}$ & $\begin{array}{l}1.67 \text { SFA g/ } \\
\text { day }(5.5 \%) \text { in } \\
\text { adults }\end{array}$ & - & - \\
\hline & & & & & Voluntary & $\begin{array}{l}0.47 \text { SFA g/ } \\
\text { day(1.50\%) in } \\
\text { adults }\end{array}$ & - & - \\
\hline \multirow[t]{3}{*}{$\begin{array}{l}\text { Leroy et al. } \\
\text { (2016) [32] }\end{array}$} & \multirow[t]{3}{*}{ Epidemiological } & \multirow[t]{3}{*}{$1 \mathrm{y}$} & \multirow[t]{3}{*}{$\begin{array}{l}\text { F\&V, bread, meat, } \\
\text { fish, sandwiches, } \\
\text { sauces }\end{array}$} & \multirow{2}{*}{$\begin{array}{l}\text { Strong } \\
\text { reformulation } \\
\text { based on the } \\
\text { Choices } \\
\text { Programme } \\
\text { criteria }\end{array}$} & \multirow[t]{2}{*}{ Mandatory } & \multirow[t]{2}{*}{$\begin{array}{l}14.8 \% \text { daily } \\
\text { SFA intake }\end{array}$} & $\begin{array}{l}\text { Fatal CVD/Strokes } \\
\text { deaths averted: } \\
1339\end{array}$ & - \\
\hline & & & & & & & $\begin{array}{l}\text { Cancer deaths } \\
\text { averted: } 558\end{array}$ & - \\
\hline & & & & $\begin{array}{l}\text { Mild reformulation } \\
\text { based on the } \\
\text { Choices } \\
\text { Programme } \\
\text { criteria }\end{array}$ & Mandatory & $\begin{array}{l}11.7 \% \text { daily } \\
\text { SFA intake }\end{array}$ & $\begin{array}{l}\text { CVD/Strokes and } \\
\text { Cancer: } 2408 \\
\text { deaths averted } \\
\text { (3.7\%) - due to } \\
\text { total reductions in } \\
\mathrm{Na} \text {, SFA and sugar } \\
\text { consumption } \\
\text { combined }\end{array}$ & - \\
\hline $\begin{array}{l}\text { Masset et al. } \\
(2016)[25]\end{array}$ & $\begin{array}{l}\text { Mathematical/ } \\
\text { statistical }\end{array}$ & $\begin{array}{l}\text { Not } \\
\text { modelled }\end{array}$ & Pizza & $\begin{array}{l}\text { Reformulation to } \\
\text { meet Nestlè } \\
\text { Nutrient Profiling } \\
\text { targets }\end{array}$ & Mandatory & $\begin{array}{l}0.3 \text { SFA g/day } \\
(1.1 \%)\end{array}$ & - & - \\
\hline \multirow[t]{2}{*}{$\begin{array}{l}\text { Pearson- } \\
\text { Stuttard et al. } \\
(2016) \text { [33] }\end{array}$} & \multirow[t]{2}{*}{ Epidemiological } & \multirow[t]{2}{*}{$10 y$} & \multirow[t]{2}{*}{ All processed food } & \multirow[t]{2}{*}{$\begin{array}{l}\downarrow 100 \% \text { (Total ban) } \\
\text { on industrial TFA }\end{array}$} & \multirow[t]{2}{*}{ Mandatory } & \multirow{2}{*}{$\begin{array}{l}\downarrow 100 \% \\
\text { industrial TFA } \\
\rightarrow \text { approx. } \\
0.4 \% \text { of daily } \\
\text { energy intake } \\
\text { from ruminant } \\
\text { TFA }\end{array}$} & $\begin{array}{l}\text { CHD deaths } \\
\text { averted or } \\
\text { postponed: } 1700 \\
(\mathrm{UI}=1619-1825)\end{array}$ & - \\
\hline & & & & & & & $\begin{array}{l}\text { LYG: } 15000 \text { (UI: } \\
\text { 13952-16,934) }\end{array}$ & - \\
\hline $\begin{array}{l}\text { Allen et al. } \\
\text { (2015) [44] }\end{array}$ & Epidemiological & $6 y$ & $\begin{array}{l}\text { All processed } \\
\text { foods }\end{array}$ & $\begin{array}{l}\downarrow 100 \% \text { (Total ban) } \\
\text { on industrial TFA }\end{array}$ & Mandatory & $\begin{array}{l}\downarrow 100 \% \\
\text { industrial TFA } \\
\rightarrow \text { approx. } \\
0.4 \% \text { of daily } \\
\text { energy intake } \\
\text { from ruminant } \\
\text { TFA }\end{array}$ & $\begin{array}{l}\text { CHD deaths } \\
\text { averted or } \\
\text { postponed: } 7200 \\
(\mathrm{UI}=3200-12,500 \text {; } \\
2.6 \%)\end{array}$ & $\begin{array}{l}7900 \text { QALYS } \\
\text { gained }(U I= \\
\text { 3000-13,900) }\end{array}$ \\
\hline \multirow[t]{3}{*}{$\begin{array}{l}\text { Combris et al. } \\
\text { (2011) [8] }\end{array}$} & \multirow[t]{3}{*}{$\begin{array}{l}\text { Mathematical/ } \\
\text { statistical }\end{array}$} & \multirow[t]{3}{*}{$\begin{array}{l}\text { Not } \\
\text { modelled }\end{array}$} & Breakfast cereals & \multirow{3}{*}{$\begin{array}{l}\text { Mild to strong } \\
\text { reformulation } \\
\text { based on food } \\
\text { nutrient } \\
\text { distribution }\end{array}$} & \multirow[t]{3}{*}{ Mandatory } & $\begin{array}{l}0.032-0.172 \text { fat } \\
\text { g/day }(1.40- \\
7.5 \%)\end{array}$ & - & - \\
\hline & & & Biscuits/ pastries & & & $\begin{array}{l}0.0162-0.061 \text { fat } \\
\text { g/day }(1.40-5.30 \%)\end{array}$ & - & - \\
\hline & & & $\begin{array}{l}\text { Bread-based } \\
\text { products }\end{array}$ & & & $\begin{array}{l}0.009-0.229 \text { fat g/day } \\
(0.40-9.90 \%)\end{array}$ & - & - \\
\hline $\begin{array}{l}\text { Temme et al. } \\
\text { (2011) [11] }\end{array}$ & $\begin{array}{l}\text { Mathematical/ } \\
\text { statistical }\end{array}$ & $\begin{array}{l}\text { Not } \\
\text { modelled }\end{array}$ & $\begin{array}{l}\text { Potato-products, } \\
\text { bread, pastry, cakes } \\
\text { and biscuits } \\
\text { (excluding foods } \\
\text { made with butter); } \\
\text { (meat) snacks and } \\
\text { salads, fats and } \\
\text { margarines }\end{array}$ & $\begin{array}{l}\downarrow 300 \% \text { TFA in } \\
\text { potato products } \\
\text { for frying; } 33 \% \text { in } \\
\text { bread; } 75 \% \text { in } \\
\text { pastry, cakes and } \\
\text { biscuits; } 67 \% \text { for } \\
\text { meat snacks and } \\
\text { salads }\end{array}$ & Mandatory & $\begin{array}{l}0.4 \text { TFA g/day }(21.1 \% \text {, } \\
\text { UI }=0.35-0.45)\end{array}$ & - & - \\
\hline \multirow[t]{2}{*}{$\begin{array}{l}\text { Roodenburg } \\
\text { et al. (2009) } \\
\text { [27] }\end{array}$} & \multirow[t]{2}{*}{$\begin{array}{l}\text { Mathematical/ } \\
\text { statistical }\end{array}$} & \multirow[t]{2}{*}{$\begin{array}{l}\text { Not } \\
\text { modelled }\end{array}$} & \multirow[t]{2}{*}{ All packaged foods } & \multirow[t]{2}{*}{$\begin{array}{l}\text { Reformulation set } \\
\text { to meet Choices } \\
\text { Programme criteria }\end{array}$} & \multirow[t]{2}{*}{ Mandatory } & $\begin{array}{l}40 \% \text { SFA daily intake } \\
\text { (32\% adjusting for } \\
\text { energy } \\
\text { compensation) }\end{array}$ & - & - \\
\hline & & & & & & $\begin{array}{l}63 \% \text { TFA daily intake (58\% } \\
\text { adjusting for energy } \\
\text { compensation) }\end{array}$ & - & - \\
\hline
\end{tabular}




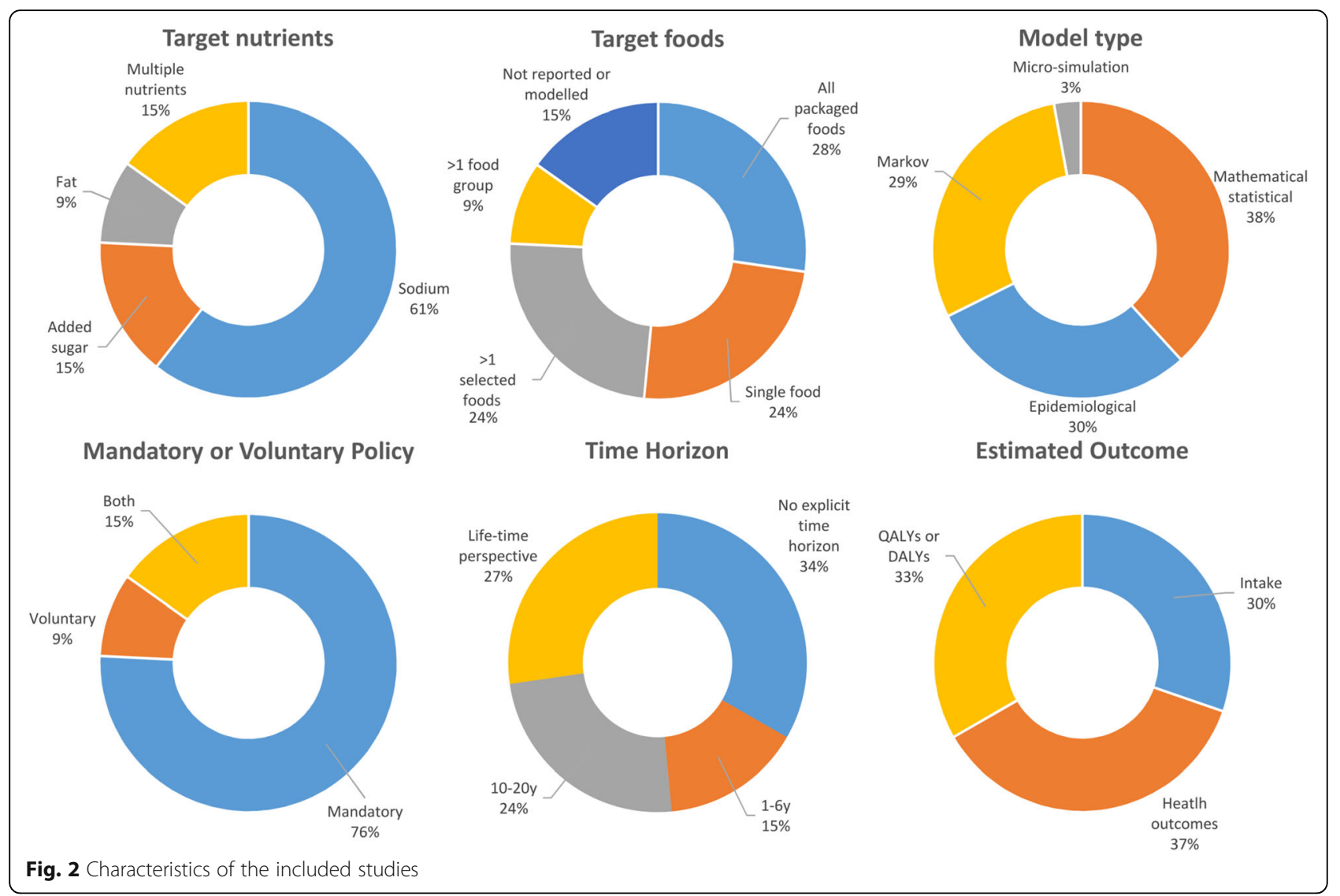

Most studies reported the number of deaths averted, life year gains and quality of life measures (QALYs or DALYs) in absolute values. This limited between-study comparisons, since results were sensitive to the time horizon of the analysis, the size of the modelled population, and baseline risk factors, which varied across studies. In the studies for which it was possible to calculate gains per 100,000 population, reductions in the sodium content of foods resulted in an increase between 265 QALYs and 12,783 QALYs [28-30], or in a reduction between 6.35 and 1452 DALYs [23, 24, 36]. Although based on less studies, results seem to be consistent across models, showing a positive association between sodium reductions and QALYs (DALYs) gained (lost).
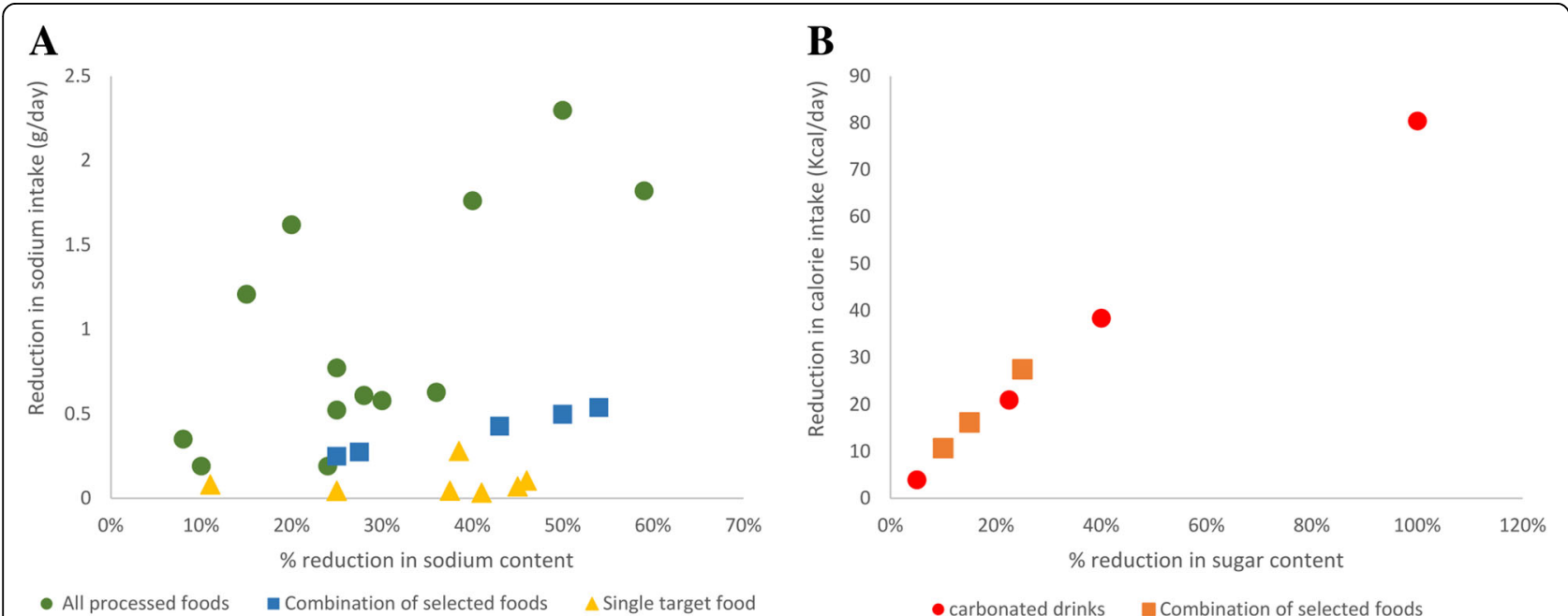

Fig. 3 Relationship between amounts reformulated and individual intakes of sodium and sugar. Scatter plot of studies reporting the effects of \% reductions in the nutrient content of food on sodium intake ( $\mathrm{g} /$ day, panel $\mathbf{a}$ ), and energy intake for sugar reformulations (Kcal/day, panel b) 


\section{Interventions to reduce intake of sugar}

Reformulation to reduce sugar intake targeted Sugar Sweetened Beverages (SSBs) alone [46-49], or other sets of foods ranging from pizza to all processed foods $[8$, 42-45, 50] (Tables 2).

Similarly to sodium strategies, although based on fewer studies, reductions in energy intake (Kcal per day) for SSBs are consistent across studies and proportional to the amount of sugar reformulated (Fig. 3, panel B). For example, Briggs et al. estimate that a reduction in the sugar content of SSBs by 5 and 23\% would reduce calorie intake by about $4 \mathrm{Kcal}$ and $21 \mathrm{Kcal}$ per day [47]. Likewise, when cutting 10 to $25 \%$ of sugar content in selected sugar-dense foods, Yeung et al. estimated a reduction in energy intake from 11 to $27 \mathrm{Kcal}$ per day [50].

Few studies modelled the impact of reformulating sugar on health outcomes. Prevalence of obesity is estimated to be reduced in a range between 0.2 and $4 \%$ [46-48], whereas one study estimated a reduction in type 2 diabetes between 5.8 and 31.1 incident cases per 100,000 persons [47]. A further study estimated that a broader reformulation policy, designed to comply with the Dutch Choices Program for selected processed foods, would yield a reduction in mortality caused by chronic disease between 3.7 and 5.5\% [44]. Lastly, no

\begin{tabular}{|c|c|c|c|c|c|c|c|c|c|}
\hline & $\begin{array}{l}\text { Problem, } \\
\text { objective } \\
\text { and scope }\end{array}$ & $\begin{array}{l}\text { Intervention } \\
\text { and } \\
\text { comparator }\end{array}$ & $\begin{array}{c}\text { Time } \\
\text { horizon }\end{array}$ & Parameters & $\begin{array}{l}\text { Relevant } \\
\text { outcomes }\end{array}$ & Uncertainty & $\begin{array}{l}\text { Internal and } \\
\text { External } \\
\text { Validation }\end{array}$ & $\begin{array}{l}\text { Face } \\
\text { Validity }\end{array}$ & $\begin{array}{l}\text { Transparency } \\
\text { and flexibility }\end{array}$ \\
\hline Briggs et al. (2017) & $\theta$ & 0 & $\otimes$ & 0 & 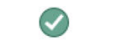 & 0 & (1) & $\theta$ & Q \\
\hline Cogswell et al. (2017) & 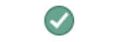 & 0 & $\otimes$ & 0 & $\otimes$ & (1) & 0 & $\otimes$ & ○ \\
\hline Yeung et al. (2017) & 0 & 0 & $\otimes$ & 0 & $\otimes$ & (1) & 0 & $\otimes$ & (1) \\
\hline Masset et al. (2016) & 0 & 0 & $\otimes$ & (1) & $\otimes$ & $\otimes$ & 0 & $\otimes$ & 0 \\
\hline Wilson et al. (2016) & ○ & ○ & ○ & (1) & $\odot$ & ○ & 0 & 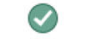 & 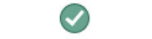 \\
\hline Food and Drink Industry Ireland (2016) & 0 & ( & $\boldsymbol{\otimes}$ & 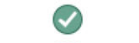 & $\otimes$ & (1) & 0 & (1) & $\boldsymbol{\otimes}$ \\
\hline Leroy et al. (2016) & $\theta$ & 0 & $\otimes$ & (1) & $\theta$ & $\otimes$ & $\otimes$ & 0 & 0 \\
\hline Ma et al. (2016) & 0 & (1) & (1) & 0 & 0 & (1) & (1) & 0 & 0 \\
\hline Nghiem et al. (2016) & 0 & 0 & 0 & 0 & 0 & 0 & 0 & 0 & 0 \\
\hline Choi et al. (2016) & 0 & 0 & (1) & 0 & 0 & (1) & (1) & 0 & 0 \\
\hline Pearson-Stuttard et al. (2016) & 0 & (1) & (1) & (1) & 0 & 0 & 0 & $\theta$ & 0 \\
\hline Gillespie et al. (2015) & 0 & (1) & (1) & ○ & $\theta$ & $\theta$ & 0 & $\theta$ & $\theta$ \\
\hline Bruins et al. (2015) & $\theta$ & $\odot$ & (1) & ○ & 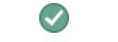 & $\otimes$ & $\otimes$ & 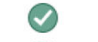 & ○ \\
\hline Wilcox et al. (2015) & 0 & (1) & (1) & (1) & 0 & (1) & 0 & 0 & (1) \\
\hline Hendriksen et al. (2015) & 0 & 0 & $\boldsymbol{\otimes}$ & 0 & $\otimes$ & $\otimes$ & 0 & $\otimes$ & 0 \\
\hline Dötsch-Klerk et al. (2015) & 0 & (1) & $\otimes$ & (1) & $\otimes$ & $\otimes$ & $\otimes$ & $\otimes$ & $\theta$ \\
\hline Nghiem et al. (2015) & $\theta$ & 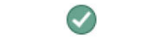 & $\theta$ & $\odot$ & 0 & $\theta$ & $\theta$ & 0 & $\theta$ \\
\hline Allen et al. (2015) & ( ) & $\theta$ & (1) & $\odot$ & $\odot$ & $\theta$ & $\theta$ & (1) & 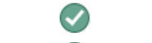 \\
\hline Hendriksen et al. (2014) & 0 & (1) & 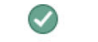 & 0 & 0 & (1) & $\boldsymbol{\otimes}$ & $\odot$ & ○ \\
\hline Mason et al. (2014) & () & (1) & $\odot$ & $\theta$ & $\theta$ & (1) & 0 & (1) & $\odot$ \\
\hline Collins et al.. (2014) & 0 & 0 & (1) & (1) & 0 & 0 & 0 & 0 & 0 \\
\hline Konfino et al. (2013) & 0 & (1) & (1) & (1) & 0 & $\otimes$ & 0 & (1) & (1) \\
\hline Bertram et al. (2012) & (1) & 0 & $\otimes$ & $\otimes$ & $\theta$ & $\otimes$ & $\otimes$ & (1) & $\otimes$ \\
\hline Cobiac et al. (2012) & $\theta$ & 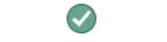 & 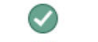 & 0 & 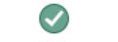 & 0 & $\boldsymbol{\otimes}$ & (1) & $\diamond$ \\
\hline Temme et al. (2011) & 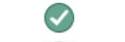 & 0 & $\boldsymbol{\otimes}$ & 0 & $\otimes$ & (1) & 0 & $\otimes$ & ○ \\
\hline Combris et al. (2011) & 0 & (1) & $\boldsymbol{\otimes}$ & (1) & $\otimes$ & $\otimes$ & 0 & $\boldsymbol{\otimes}$ & $\odot$ \\
\hline Hendriksen et al. (2011) & (1) & (1) & $\otimes$ & (1) & (1) & $\otimes$ & (1) & $\otimes$ & $\theta$ \\
\hline Cobiac et al. (2010) & 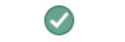 & 0 & 0 & 0 & 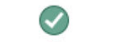 & 0 & $\otimes$ & 0 & ( \\
\hline Smith-Spangler et al. (2010) & $\theta$ & (1) & 0 & 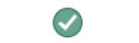 & $\theta$ & $\odot$ & ( & (1) & 0 \\
\hline Roodenburg et al. (2009) & ○ & $\otimes$ & $\otimes$ & $\theta$ & $\otimes$ & (1) & 0 & $\otimes$ & (1) \\
\hline Rubinstein et al.. (2009) & 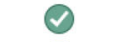 & 0 & 0 & (1) & 0 & (1) & $\otimes$ & 0 & $\odot$ \\
\hline Husøy et al. (2008) & (1) & (1) & $\otimes$ & ○ & $\otimes$ & $\otimes$ & 0 & $\otimes$ & ○ \\
\hline \multirow[t]{5}{*}{ Murray et al. (2003) } & 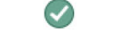 & (1) & 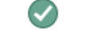 & $\theta$ & $\theta$ & $\theta$ & $\otimes$ & (1) & (1) \\
\hline & 0 & high score & & & & & & & \\
\hline & (1) & moderate score & & & & & & & \\
\hline & $\otimes$ & low score & & & & & & & \\
\hline & - & Not applicable & & & & & & & \\
\hline
\end{tabular}


studies were found that estimated the impact of reformulation on health related quality of life measures.

\section{Interventions to reduce intake of fat}

Studies addressing reformulation of SFA or TFA are limited. Temme et al., estimated that a broad intervention cutting TFA on a set of products would lower trans-fat consumption by $0.4 \mathrm{~g} /$ day $(21.1 \%, \mathrm{UI}=0.35,0.45)$ (Table 3) [11]. In addition, two studies estimated that banning all industrial TFA from processed foods would avert 1700 to 7200 deaths and generate a gain of 7900 QALYs $[51,52]$.

Other interventions modelling broader multi-nutrient reformulations for different target products found percentage reductions in SFA consumption to be in a range between 1.1 and 40\% [43-45] (Table 2). One single study estimated the effect of fat reformulation on mortality, showing that if all producers in the food industry complied with the International Choices Programme [27], SFA consumption would reduce by $15 \%$, which in turn would reduce deaths by $3 \%$ (1339 deaths from cardiovascular diseases and 558 deaths from cancer) [44].

\section{Quality of studies}

Most of the studies (64\%) scored high in at least 50\% of the considered assessment elements, whereas $9 \%$ of studies had mainly moderate scores and $27 \%$ received low scores. Study quality was generally satisfactory in the criteria relating to the description of the problem, the research objective and the scope (91\% of the included studies); the transparency and provision of technical documentation (79\%); the type of outcomes included (67\%). However, studies generally scored poorly when assessing the adequacy of the time horizon ( $42 \%$ low and $27 \%$ moderate scores), the management and reporting of uncertainty in the model parameters (30\% low, 33\% moderate) and the internal and external validation (38\% low, 17\% moderate). Lastly, only $45 \%$ of the studies scored high in face validity that is their results were considered credible and realistic (Fig. 4). Particularly, studies were considered to have high face validity if they duly and credibly took into account all aspects of the decision problem, including the technical feasibility of the reformulation scenarios, and all the causal steps linking the intervention to the outcomes of interests (e.g. the reactions of the consumers and manufacturers to the intervention).

\section{Discussion}

The present systematic review collected and synthetized the results of simulation models estimating the effect of reformulation policies to improve population diets. Most of the studies focused on sodium, which is not unexpected since sodium reduction strategies have been a policy priority for much longer compared to those for sugar and fats. All models predicted positive outcomes; however, between-study comparability was limited, especially for health outcomes and health related quality of life measures. In addition, no studies estimated long-term consequences on QALYs or DALYs for sugar, TFA, SFA, or broader multi-nutrient reformulations. More research is needed to evaluate the potential impact of reformulation strategies in achieving national and international consumption targets, and ultimately improving public health.

In addition, more evidence is required on the relative effectiveness and cost-effectiveness of reformulation compared to alternative public health nutrition interventions (e.g. food taxes, public media campaigns or food labelling).

The effectiveness of reformulation strategies on public health is the result of a complex causal chain that, besides biological factors linking intake to health outcomes, it also includes technical/industrial aspects, marketplace dynamics and consumer reactions. The incorporation of these aspects relates to the face validity of the model, that is defined as the extent to which a model plausibly represents the diseases, settings, populations, interventions, and outcomes it is intended to analyze [21].

For $30 \%$ of the included studies, strategies were not clearly informed by aspects related to the technical feasibility of reformulation, including the essential requirements to preserve shelf-life, volume and palatability. However, since the amounts of nutrients substituted in the scenarios directly affect the estimated impact on intake and health, the nature and the intensity of reformulation strategies should always be defined based on real observed reductions from on-going initiatives, or informed by reliable evidence or expert opinions.

In addition, reformulation can alter the sensory attributes of food products and influence consumer liking [54, 55]. This in turn may trigger unattended behaviors including the consumption of more public health sensitive nutrients, or simply more calories. Nonetheless, only two of the included studies explicitly modelled how consumer reactions might affect the policy effects. Choi et al. estimated that the beneficial effects of a mandatory reformulation policy would remain even if the use of discretionary salt increased by $15 \%$ [31]. However, such threshold percentage may be lower for less pervasive or voluntary reformulation policies. Roodenburg et al. tested the results of the model against the possibility that consumers maintained the same caloric intake by eating more food [45]. They found that, after this adjustment, differences in intakes for energy, SFA, TFA, sodium and sugar would be smaller. In addition, while there is evidence that consumers gradually adapt to changes in the salt content of foods $[56,57]$ and that small salt reductions in certain products cannot be detected and do not affect acceptability or consumption $[34,58,59]$, this is more controversial for fat [60] and sugar [61]. 
With respect to marketplace dynamics, unsurprisingly, in the few studies where both mandatory and voluntary interventions were modelled, mandatory scenarios were always found to be more effective. Nevertheless, such differences may partly reflect various assumptions around intervention phase-in periods [29], or the considered rate of adherence from the industry. For example, when allowing for the possibility that consumers switched to non-reformulated products, Choi et al. estimate that significant reductions in CVD mortality would occur only if more than $65 \%$ of products in the market met the reduction criteria [31].

Anticipating how food manufacturers and consumers will react to a reformulation initiative are core components of models, so that lack of consideration for these aspects may undermine the credibility of the model results. Since there may be little data on these parameters at the time of the assessment, studies should clearly report and justify the underlying assumptions made in their models, and conduct extensive sensitivity analysis to test the robustness of results at different levels of industry uptake and consumers reactions. In addition, in some cases, real world sources can be used to model more realistic scenarios. A study by Temme et al. assessing the impact on intake of foods reporting a health logo estimated the expected consumption rate of healthy and unhealthy products, by looking at real market shares of products with a healthy logo over the total purchases in each food category [62].

The time horizon considered in reformulation models should be long enough to account for all relevant consequences of the interventions, including the long-term health effects of improved dietary patterns. In addition, many aspects of the reformulation models can vary over time including the industry uptake of reformulated products, consumption habits and preferences, and secular epidemiological trends in non-communicable diseases. In this review, $40 \%$ of the studies did not explicitly model the effects of the interventions over time and focused on simulating how cross-sectional nutrient intake data would change if foods were reformulated. Although consistent with their declared research questions, these studies do not estimate how the modelled interventions will dynamically affect food purchases, intake, and ultimately health outcomes. The dynamic nature of public health interventions and the presence of complex, interdependent factors have already been pointed out in the literature [63-65] calling for more methodological developments, such as the use of system dynamics modelling to incorporate time dependencies [66].

Besides face validity, other standard steps of validation apply to reformulation models [21]. Internal validity should be verified by demonstrating that the model behaves as intended and has been implemented correctly. In addition, whenever possible, the simulated model outcomes should be confronted with real-world event data. External validation involves that the entire model or any of its components are verified by confronting the forecasted estimates with actual event data. For example, epidemiological studies or trial data could be used to verify the correctness of the simulated incidence of non-communicable diseases in the absence of reformulation. In addition, models should prove to be able to correctly predict future outcomes for the specific setting, population and intervention of interest. Therefore, when possible, the results of the model should be compared ex-post with the real-world outcomes, should the modelled intervention be implemented as planned. However, assessing the predictive ability of a model for medium and long term effects such as health and quality of life is challenging, as it would require longitudinal data with a rigorous counterfactual scenario [67]. While this type of evidence is often missing, partial validation could be achieved by assessing the goodness of models in predicting intermediary effects such as intakes or surrogate health outcomes (e.g. hypertension).

External and predictive validation are critical for simulation models as their main purpose is primarily to help decision makers anticipate what will occur after introducing a certain policy [68]. Therefore, models should be transparent on how validation is verified. However, only one third of the studies used previously validated models or reported methods used to validate their own models. A more careful consideration of validation would improve study quality and increase trust and confidence in models to inform policy-making.

This review reported the potential effects of reformulation policies on intake and health and proposed an ad-hoc tool to assess the quality of modelling studies on reformulation. .

Nonetheless, a number of limitations are outlined: studies were very different in the way policy effects were modelled and reported so that the provided ranges of effectiveness should be considered with caution; this heterogeneity is a reflection of the lack of guidelines and standardized methods for the evaluation of public health interventions in general. Methods for the evaluation of healthcare interventions have indeed existed for several years [63, 68-72], but these have mainly been applied to more narrowly-defined 'clinical' interventions, such as drugs, devices and medical procedures [64]. In contrast, approaches in the field of nutrition and public health are not framed by common objectives, shared methods and/ or a strong regulatory environment. Therefore, the establishment of an agreed framework specifying best modelling practices is needed to improve the methodological and reporting quality, as well as the comparability of studies evaluating public health interventions in general and nutrition interventions in particular. 
Finally, the number of available studies and the described heterogeneity did not allow to perform subgroup analyses to verify whether specific model features or study quality impact their results. Particularly, in this study, we did not assess existing sources of variations in the effectiveness of reformulation and other nutrition interventions between and within countries, including biological, cultural, socio-economic and institutional factors. Future work may explore the contribution of these aspects on each link of the causal chain from intervention to public health outcomes. Explaining cross-country variations may help to understand which factors are favoring or hindering the effectiveness of nutrition policies at the global level, and their role in reducing the burden of non-communicable diseases. Explaining within-country variations may contribute to incorporate equity considerations in public decision making about nutrition policies at the national level.

\section{Conclusions}

Reformulation policies have the potential to improve diets and population health. Evidence is stronger for sodium interventions, but far less conclusive for sugar and fat reformulations. Mathematical models are valuable tools to predict policy effects, although comparability is often limited by different study designs, assumptions and reporting quality. More homogeneous designs and assumptions, combined with the validation of model results and extensive scenario analysis to evaluate the relevance of specific policy features, would improve model credibility and provide policy-makers with useful insights to design evidence-based nutrition policies.

\section{Additional files}

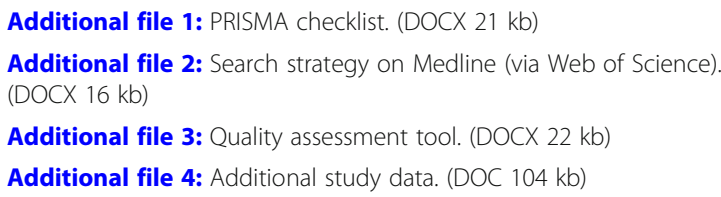

\section{Abbreviations}

AMI: Acute myocardial infarction; BMI: Body mass index; CHF: Congestive heart failure; CVD: Cardiovascular diseases; DALY: Disability adjusted life years; LYG: Life-years gained; QALY: Quality adjusted life years; SFA: Saturated fatty acids; SSB: sugar sweetened beverage; T2D: Type 2 diabetes; TFA: Trans-fatty acids; UI: Uncertainty interval

\section{Acknowledgments}

Authors thank Dr. Zsuzsa Hutton (Nestlé Research Center, Lausanne, Switzerland) for her support in the organization of the project and for the revision of the final manuscript.

\section{Funding}

These findings are the results of work supported by Nestec SA. The views expressed in this paper are those of the authors, and no official endorsement by Nestec SA is intended or should be inferred.
Availability of data and materials

All data generated or analysed during this study are included in this published article [and its supplementary information files].

\section{Authors' contributions}

The authors responsibilities were as follows: CF, PD, and GF designed research. CF and FP conducted research and analyzed data; CF wrote the paper; CF, PD, GF and LD had primary responsibility for the final content. All authors have read and approved the final manuscript

Ethics approval and consent to participate

Not applicable.

Consent for publication

Not applicable.

\section{Competing interests}

the authors declare that they have no competing interests.

\section{Publisher's Note}

Springer Nature remains neutral with regard to jurisdictional claims in published maps and institutional affiliations.

\section{Author details}

${ }^{1}$ CeRGAS (Centre for Research on Health and Social Care Management), SDA Bocconi School of Management, Milan, Italy. ${ }^{2}$ Nestlé Research Center, Nestec SA, Lausanne, Switzerland. ${ }^{3}$ Department of Policy Analysis and Public Management, Bocconi University, Milan, Italy.

Received: 13 March 2018 Accepted: 12 December 2018

Published online: 07 January 2019

\section{References}

1. Mozaffarian D, Clarke R. Quantitative effects on cardiovascular risk factors and coronary heart disease risk of replacing partially hydrogenated vegetable oils with other fats and oils. Eur J Clin Nutr. 2009;63(Supplement 2):S22-33. https://doi.org/10.1038/sj.ejcn.1602976.

2. Mozaffarian D, Katan MB, Ascherio A, Stampfer MJ, Willett WC. Trans fatty acids and cardiovascular disease. NEJM. 2006;15:1601-13. https://doi.org/10. 1056/NEJMra054035

3. World Health Organisation. Diet, Nutrition and the prevention of chronic diseases. 2003. http://www.who.int/dietphysicalactivity/publications/trs916/ en/. Accessed 18 Apr 2016

4. World Cancer Research Fund / American Institute for Cancer Research. Food, nutrition and the prevention of cancer: a global perspective. Washington, D.C: AlCR; 2007. https://doi.org/10.1007/s12082-007-0105-4.

5. Key TJ, Schatzkin A, Willett WC, Allen NE, Spencer EA, Travis RC. Diet, nutrition and the prevention of type 2 diabetes. Public Health Nutr. 2004;7: 147-65. https://doi.org/10.1079/PHN2003586.

6. He FJ, MacGregor GA, Boon C. A comprehensive review on salt and health and current experience of worldwide salt reduction programmes. J Hum Hypertens. 2009;23:363-84

7. Public Health England. Sugar reduction: Achieving the $20 \%$ - GOV.UK. London; 2017. https://www.gov.uk/government/publications/sugarreduction-achieving-the-20. Accessed 24 May 2017.

8. Combris P, Goglia R, Henini M, Soler LG, Spiteri M. Improvement of the nutritional quality of foods as a public health tool. Public Health. 2011;125: 717-24. https://doi.org/10.1016/j.puhe.2011.07.004.

9. Griffith $\mathrm{R}, \mathrm{O}^{\prime}$ Connell $\mathrm{M}$, Smith $\mathrm{K}$. The importance of product reformulation versus consumer choice in improving diet quality. Economica. 2017;84:3453. https://doi.org/10.1111/ecca.12192.

10. Scott C, Hawkins B. Knai C. Soc Sci Med: Food and beverage product reformulation as a corporate political strategy; 2017.

11. Temme EHM, Millenaar IL, Van Donkersgoed G, Westenbrink S. Impact of fatty acid food reformulations on intake of Dutch young adults. Acta Cardiol. 2011:66:721-8. https://doi.org/10.2143/AC.66.6.2136955.

12. Webster J, Trieu K, Dunford E, Hawkes C. Target salt 2025: a global overview of national programs to encourage the food industry to reduce salt in foods. Nutrients. 2014;6:3274-87. https://doi.org/10.3390/nu6083274. 
13. Public Health England. Guidelines on reducing sugar in food published for industry - GOV.UK. 2017. https://www.gov.uk/government/news/guidelineson-reducing-sugar-in-food-published-for-industry. Accessed 28 May 2017.

14. Grieger JA, Johnson BJ, Wycherley TP, Golley RK. Evaluation of simulation models that estimate the effect of dietary strategies on nutritional intake: a systematic review. J Nutr. 2017;147:908-31. https://doi.org/10.3945/jn.116. 245027.

15. Mcdaid D, Sassi F, Merkur S, Sassi F, Mcdaid D. Promoting health, preventing disease: is there an economic case? Policy summary 6. 2013. http://www. euro.who.int/_data/assets/pdf_file/0004/235966/e96956.pdf. Accessed 5 Sep 2017.

16. Buxton MJ, Drummond MF, Van Hout BA, Prince RL, Sheldon TA, Szucs T, et al. Modelling in economic evaluation: an unavoidable fact of life. Health Econ. 1997;6:217-27. https://doi.org/10.1002/(SICI)1099-1050(199705)6: 3<217::AID-HEC267>3.0.CO;2-W

17. Shamseer L, Moher D, Clarke M, Ghersi D, Liberati A, Petticrew M, et al. Preferred reporting items for systematic review and meta-analysis protocols (PRISMA-P) 2015: elaboration and explanation. BMJ. 2015;349:97647.

18. Moher D, Shamseer $L$, Clarke M, Ghersi D, Liberati A, Petticrew M, et al, Preferred reporting items for systematic review and meta-analysis protocols (PRISMA-P) 2015 statement. Syst Rev. 2015;4:1. https://doi.org/10.1186/20464053-4-1.

19. Moher D, Liberati A, Tetzlaff J, Altman DG. PRISMA group. Preferred Reporting Items for Systematic Reviews and Meta-Analyses: The PRISMA Statement J Clin Epidemiol. 2009;62:1006-12.

20. Hope SF, Webster J, Trieu K, Pillay A, leremia M, Bell C, et al. A systematic review of economic evaluations of population-based sodium reduction interventions. PLoS One. 2017;12:e0173600. https://doi.org/10.1371/journal. pone.0173600

21. Jaime Caro J, Eddy DM, Kan H, Kaltz C, Patel B, Eldessouki R, et al. Questionnaire to assess relevance and credibility of modeling studies for informing health care decision making: an ISPOR-AMCP-NPC good practice task force report. Value Heal. 2014;17:174-82. https://doi.org/10.1016/j.jval. 2014.01.003

22. Murray CJ, Lauer JA, Hutubessy RC, Niessen L, Tomijima N, Rodgers A, et al. Effectiveness and costs of interventions to lower systolic blood pressure and cholesterol: a global and regional analysis on reduction of cardiovasculardisease risk. Lancet. 2003;361:717-25. https://doi.org/10.1016/S01406736(03)12655-4

23. Rubinstein A, García Martí S, Souto A, Ferrante D, Augustovski F. Generalized cost-effectiveness analysis of a package of interventions to reduce cardiovascular disease in Buenos Aires. Argentina Cost Eff Resour Alloc. 2009;7:10. https://doi.org/10.1186/1478-7547-7-10.

24. Bruins MJ, Dötsch-Klerk M, Matthee J, Kearney M, van Elk K, Weber P, et al. A modelling approach to estimate the impact of sodium reduction in soups on cardiovascular health in the Netherlands. Nutrients. 2015;7:8010-9. https://doi.org/10.3390/nu7095375.

25. Wilcox ML, Mason H, Fouad FM, Rastam S, al Ali R, Page TF, et al. Costeffectiveness analysis of salt reduction policies to reduce coronary heart disease in Syria, 2010-2020. Int J Public Health. 2015;60(Suppl 1):S23-30. https://doi.org/10.1007/s00038-014-0577-3.

26. Hendriksen MAH, Verkaik-Kloosterman J, Noort MW, van Raaij JMA. Nutritional impact of sodium reduction strategies on sodium intake from processed foods. Eur J Clin Nutr. 2015;69:805-10. https://doi.org/10.1038/ejcn.2015.15.

27. Dötsch-Klerk M, Jansen L. The choices programme: a simple, front-of-pack stamp making healthy choices easy. Asia Pac J Clin Nutr. 2008;17(SUPPL. 1): 383-6.

28. Nghiem N, Blakely T, Cobiac LJ, Pearson AL, Wilson N. Health and economic impacts of eight different dietary salt reduction interventions. PLoS One 2015;10:e0123915. https://doi.org/10.1371/journal.pone.0123915.

29. Wilson N, Nghiem N, Eyles H, Mhurchu CN, Shields E, Cobiac L, et al. Modeling health gains and cost savings for ten dietary salt reduction targets. Nutr J. 2016;15:44. https://doi.org/10.1186/s12937-016-0161-1.

30. Nghiem N, Blakely T, Cobiac LJ, Cleghorn CL, Wilson N. The health gains and cost savings of dietary salt reduction interventions, with equity and age distributional aspects. BMC Public Health. 2016;16:423. https://doi.org/10. 1186/s12889-016-3102-1.

31. Choi SE, Brandeau ML, Basu S. Expansion of the National Salt Reduction Initiative: a mathematical model of benefits and risks of population-level sodium reduction. Med Decis Mak. 2016;36:72-85. https://doi.org/10.1177/ 0272989X15583846.
32. Smith-Spangler CM, Juusola JL, Enns EA, Owens DK, Garber AM. Population strategies to decrease sodium intake and the burden of cardiovascular disease. Ann Intern Med. 2010;152:481. https://doi.org/10.7326/0003-4819152-8-201004200-00212.

33. Cogswell ME, Patel SM, Yuan K, Gillespie C, Juan W, Curtis CJ, et al. Modeled changes in US sodium intake from reducing sodium concentrations of commercially processed and prepared foods to meet voluntary standards established in North America: NHANES. Am J Clin Nutr. 2017:106:530-40.

34. Cobiac $L$, Vos T, Veerman JL. Cost-effectiveness of interventions to reduce dietary salt intake. Heart. 2010;96:1920-1925. https://doi.org/10.1136/hrt.2010. 199240

35. Bertram, MY, Steyn, K, Wentzel-Viljoen, E, Tollman, S, Hofman, KJ. Reducing the sodium content of high-salt foods: effect on cardiovascular disease in South Africa. South African Medical Journal. 2012:102(9):743-745.

36. Cobiac L, Magnus A, Lim S, Barendregt JJ, Carter R, Vos T. Which interventions offer best value for money in primary prevention of cardiovascular disease? PLoS One. 2012;7:e41842. https://doi.org/10.1371/ journal.pone.0041842.

37. Konfino J, Mekonnen TA, Coxson PG, Ferrante D, Bibbins-Domingo K. Projected impact of a sodium consumption reduction initiative in Argentina: an analysis from the CVD policy model - Argentina. PLoS One. 2013;8:e73824. https://doi.org/10.1371/journal.pone.0073824.

38. Hendriksen MA, Hoogenveen RT, Hoekstra J, Geleijnse JM, Boshuizen HC, van Raaij JM. Potential effect of salt reduction in processed foods on health. Am J Clin Nutr. 2014;99:446-53. https://doi.org/10.3945/ajcn.113.062018.

39. Mason H, Shoaibi A, Ghandour R, O'Flaherty M, Capewell S, Khatib R, et al. A cost effectiveness analysis of salt reduction policies to reduce coronary heart disease in four eastern Mediterranean countries. PLoS One. 2014;9: e84445. https://doi.org/10.1371/journal.pone.0084445.

40. Collins M, Mason H, O'Flaherty M, Guzman-Castillo M, Critchley J, Capewell $\mathrm{S}$. An economic evaluation of salt reduction policies to reduce coronary heart disease in England: a policy modeling study. Value Health. 2014;17: 517-24. https://doi.org/10.1016/j.jval.2014.03.1722.

41. Gillespie DOS, Allen K, Guzman-Castillo M, Bandosz P, Moreira P, McGill R, et al. The health equity and effectiveness of policy options to reduce dietary salt intake in England: policy forecast. PLoS One. 2015;10:e0127927. https:// doi.org/10.1371/journal.pone.0127927.

42. Masset G, Mathias KC, Vlassopoulos A, Mölenberg F, Lehmann U, Gibney M, et al. Modeled dietary impact of pizza reformulations in US children and adolescents. PLoS One. 2016;11:e0164197. https:/doi.org/10.1371/journal.pone.0164197.

43. Food and Drink Industry Ireland. The FDII/Creme Global Reformulation Project. 2016. https://www.fooddrinkireland.ie/Sectors/FDI/FDI.nsf/vPages/ Publications fdi-creme-global-reformulation-report-27-012016! OpenDocument

44. Leroy $P$, Réquillart $V$, Soler L-G, Enderli G. An assessment of the potential health impacts of food reformulation. Eur J Clin Nutr. 2016;70:694-9. https:// doi.org/10.1038/ejcn.2015.201.

45. Roodenburg AJC, Temme EHM, Davies OH, Seidell JC. Potential impact of the choices Programme on nutrient intakes in the Dutch population. Nutr Bull. 2009:34:318-23. https://doi.org/10.1111/j.1467-3010.2009.01767.x.

46. Ma Y, He FJ, Yin Y, Hashem KM, MacGregor GA. Gradual reduction of sugar in soft drinks without substitution as a strategy to reduce overweight, obesity, and type 2 diabetes: a modelling study. lancet Diabetes Endocrinol. 2016;4:105-14. https://doi.org/10.1016/S2213-8587(15)00477-5.

47. Briggs ADM, Mytton OT, Kehlbacher A, Tiffin R, Elhussein A, Rayner M, et al. Health impact assessment of the UK soft drinks industry levy: a comparative risk assessment modelling study. Lancet Public Heal. 2017;2:e15-22. https:// doi.org/10.1016/S2468-2667(16)30037-8.

48. Hendriksen MA, Tijhuis MJ, Fransen HP, Verhagen H, Hoekstra J. Impact of substituting added sugar in carbonated soft drinks by intense sweeteners in young adults in the Netherlands: example of a benefit-risk approach. Eur J Nutr. 2011;50:41-51. https://doi.org/10.1007/s00394-010-0113-Z.

49. Husøy T, Mangschou B, Fotland T $\varnothing$, Kolset SO, Nøtvik Jakobsen H, Tømmerberg I, et al. Reducing added sugar intake in Norway by replacing sugar sweetened beverages with beverages containing intense sweeteners - a risk benefit assessment. Food Chem Toxicol. 2008;46:3099-105. https://doi.org/ 10.1016/j.fct.2008.06.013.

50. Yeung CHC, Gohil P, Rangan AM, Flood VM, Arcot J, Gill TP, et al. Modelling of the impact of universal added sugar reduction through food reformulation. Sci Rep. 2017;7:17392. 
51. Pearson-Stuttard J, Hooton W, Critchley J, Capewell S, Collins M, Mason H, et al Cost-effectiveness analysis of eliminating industrial and all trans fats in England and Wales: modelling study. J Public Health (Bangkok). 2016;39:574-82.

52. Allen K, Pearson-Stuttard J, Hooton W, Diggle P, Capewell S, O'Flaherty M. Potential of trans fats policies to reduce socioeconomic inequalities in mortality from coronary heart disease in England: cost effectiveness modelling study. BMJ. 2015;351:h4583.

53. Dötsch-Klerk M, Goossens WP, Meijer GW, van het Hof KH. Reducing salt in food; setting product-specific criteria aiming at a salt intake of $5 \mathrm{~g}$ per day. Eur J Clin Nutr. 2015;69:799-804. https://doi.org/10.1038/ejcn.2015.5.

54. Chollet M, Gille D, Schmid A, Walther B, Piccinali P. Acceptance of sugar reduction in flavored yogurt. J Dairy Sci. 2013;96:5501-11. https://doi.org/10. 3168/jds.2013-6610.

55. Hoppu U, Hopia A, Pohjanheimo T, Rotola-Pukkila M, Mäkinen S, Pihlanto A, et al. Effect of salt reduction on consumer acceptance and sensory quality of food. Foods. 2017;6:103. https://doi.org/10.3390/foods6120103.

56. Bertino M, Beauchamp GK, Engelman K. Long-term reduction in dietary sodium alters the taste of salt. The American journal of clinical nutrition. 1982;36(6):1134-1144. https://doi.org/10.1093/ajcn/36.6.1134

57. Blais CA, Pangborn RM, Borhani NO, Ferrell MF, Prineas RJ, Laing B. Effect of dietary sodium restriction on taste responses to sodium chloride: a longitudinal study. The American journal of clinical nutrition. 1986;44(2):232243. https://doi.org/10.1093/ajcn/44.2.232

58. Girgis S, Neal B, Prescott J, Prendergast J, Dumbrell S, Turner C, et al. A onequarter reduction in the salt content of bread can be made without detection. Eur J Clin Nutr. 2003;57:616-20. https://doi.org/10.1038/sj.ejcn.1601583.

59. Rodgers A, Neal B, Engelman K, Wills R. Less salt does not necessarily mean less taste. Lancet (London, England). 1999;353:1332. doi:https://doi.org/10. 1016/S0140-6736(99)01198-8.

60. Mytton O, Gray A, Rayner M, Rutter H. Could targeted food taxes improve health? J Epidemiol Community Health. 2007;61:689-94. https://doi.org/10 1136/jech.2006.047746.

61. Markey O, Lovegrove JA, Methven L. Sensory profiles and consumer acceptability of a range of sugar-reduced products on the UK market. Food Res Int. 2015;72:133-9. https://doi.org/10.1016/j.foodres.2015.03.012.

62. Temme EH, van der Voet H, Roodenburg AJ, Bulder A, van Donkersgoed G, van Klaveren J. Impact of foods with health logo on saturated fat, sodium and sugar intake of young Dutch adults. Public health nutrition. 2011;14(4): 635-644. https://doi.org/10.1017/S1368980010002089

63. Jaime Caro J, Briggs AH, Siebert U, Kuntz KM. Modeling Good Research Practices-Overview: A Report of the ISPOR-SMDM Modeling Good Research Practices Task Force-1 on Behalf of the ISPOR-SMDM Modeling Good Research Practices Task Force. https://doi.org/10.1016/j.jval.2012.06.012.

64. Weatherly H, Drummond M, Claxton K, Cookson R, Ferguson B, Godfrey C, et al. Methods for assessing the cost-effectiveness of public health interventions: Key challenges and recommendations. Health Policy (New York). 2009;93:85-92. https://doi.org/10.1016/j.healthpol.2009.07.012.

65. Kelly AMP, Mcdaid D, Ludbrook A, Powell J. Economic appraisal of public health interventions. Development. 2004:1-8. http://citeseerx.ist.psu.edu/ viewdoc/download?doi=10.1.1.476.3820\&rep=rep1\&type=pdf.

66. Homer JB, Hirsch GB. System dynamics modeling for public health: background and opportunities. Am J Public Health. 2006.

67. Mazzocchi M. Ex-post evidence on the effectiveness of policies targeted at promoting healthier diets. 2017. http://www.fao.org/3/a-i8191e.pdf.

68. Eddy DM, Hollingwoth W, Jaime Caro J, Tsevat J, McDonald KM, Wong JB. Model transparency and validation: a report of the ISPOR-SMDM modeling good research practices task Force-7. Value Heal. 2012;15:843-50.

69. Husereau D, Drummond M, Petrou S, Carswell C, Moher D, Greenberg D. Consolidated health economic evaluation reporting standards (CHEERS)-explanation and elaboration: a report of the ISPOR health economic evaluations publication guidelines task force. Value Heal. 2013;16:231-50.

70. Drummond MF, Jefferson TO. Guidelines for authors and peer reviewers of economic submissions to the BMJ. The BMJ Economic Evaluation Working Party. BMJ: British Medical Journal. 1996;313(7052):275.

71. Drummond MF, Sculpher MJ, Claxton K, Stoddart GL, Torrance GW. Methods for the Economic Evaluation of Health Care Programmes. 4th ed. Oxford: Oxford University Press; 2015.

72. Evers $\mathrm{S}$, Goossens $\mathrm{M}$, de Vet $\mathrm{H}$, van Tulder M, Ament A. Criteria list for assessment of methodological quality of economic evaluations: Consensus on Health Economic Criteria. Int J Technol Assess Health Care. 2005;21:2405. https://doi.org/10.1017/S0266462305050324

Ready to submit your research? Choose BMC and benefit from:

- fast, convenient online submission

- thorough peer review by experienced researchers in your field

- rapid publication on acceptance

- support for research data, including large and complex data types

- gold Open Access which fosters wider collaboration and increased citations

- maximum visibility for your research: over $100 \mathrm{M}$ website views per year

At BMC, research is always in progress.

Learn more biomedcentral.com/submissions 